\title{
STABILITY ANALYSIS OF UNSTEADY, NONUNIFORM BASE STATES IN THIN FILM EQUATIONS*
}

\author{
MARION DZIWNIK ${ }^{\dagger}$, MACIEK KORZEC ${ }^{\dagger}$, ANDREAS MÜNCH ${ }^{\ddagger}$, AND BARBARA \\ WAGNER $^{\dagger}$
}

\begin{abstract}
We address the linear stability of unsteady and nonuniform base states within the class of mass conserving free boundary problems for degenerate and nondegenerate thin film equations. Well-known examples are the finger-instabilities of growing rims that appear in retracting thin solid and liquid films. Since the base states are time dependent and do not have a simple traveling wave or exact self-similar form, a classical eigenvalue analysis fails to provide the dominant wavelength of the instability. However, the initial fronts evolve on a slower time-scale than the typical perturbations. We exploit this time-scale separation and develop a multiple-scale approach for this class of stability problems. We show that the value of the dominant wavelength is rapidly attained once the base state has entered an asymptotically self-similar form. We note that this value is different from the one obtained by the linear stability analysis with "frozen modes," frequently found in the literature. Furthermore, we show that for the present class of stability problems that the dispersion relation is linear in the long wave limit, which is in contrast to many other instability problems in thin film flows.
\end{abstract}

Key words. multiple-scale methods, stability analysis, rim instability, free boundaries, dewetting films

AMS subject classifications. 76M45, 76E17, 35B40, 35C20

DOI. $10.1137 / 130943352$

1. Introduction. Linear stability analysis is one of the most important tools for predicting pattern formation in many phenomena in nature and technological processes. Historically, this theory has been developed to explain some of the most fundamental instabilities in hydrodynamics and other fields, such as Rayleigh-Bénard convection or vortices in Taylor-Couette flow, to name only two examples. The basic idea is to assume that the emergence of a pattern is initiated by infinitesimally small perturbations of a typically uniform base state, which then evolve according to a linearized system of model equations. If the shape of the base state is constant in time, the coefficients of the linearized problem are time independent, and the general solution can be constructed, in principle from the knowledge of the spectrum of the operator that describes the linearized model. If the spectrum extends into the right half of the complex plane, some components of a generic perturbation (arising in practice as noise, for example) will grow, typically exponentially, thus driving the system into a new state. Moreover, the part of the spectrum with the largest real part will eventually dominate the evolving features and, e.g., determine the wavelength of periodic patterns. This approach is commonly called normal modes analysis and has shown to be an accurate method for a vast range of stability problems.

However, this concept has to be reconsidered for problems where the correspond-

*Received by the editors October 29, 2013; accepted for publication (in revised form) March 13, 2014; published electronically June 17, 2014. The authors gratefully acknowledge the support of the Federal Ministry of Education (BMBF) and the state government of Berlin (SENBWF) under the framework of the program "Spitzenforschung und Innovation in den Neuen Ländern" (grant 03IS2151).

http://www.siam.org/journals/mms/12-2/94335.html

${ }^{\dagger}$ Technische Universität Berlin, Institute of Mathematics, 10623 Berlin, Germany (dziwnik@ math.tu-berlin.de, korzec@math.tu-berlin.de, wagner@math.tu-berlin.de).

${ }^{\ddagger}$ Mathematical Institute, University of Oxford, Oxford OX1 3LB, UK (muench@maths.ox.ac.uk). 
ing eigenvalue problem has nonorthogonal eigenfunctions, i.e., the linearized operator is nonnormal. In this case, even if all of the corresponding eigenvalues, or growth rates, are negative, perturbations may still be amplified arbitrarily large. This is known as transient growth and has been established as a mechanism that can give sufficiently large amplitude corrugations to destabilize the system by exciting nonlinear effects $[6,13,16]$. In fact, it was shown in [38] that transient growth can be investigated within the framework of pseudospectra of the corresponding linear system. Since then, these ideas have been put to fruitful use in many (in)stability problems, e.g., in fingering problems for thin films $[2,17]$ and in Rayleigh-Bénard-Marangoni convection [8].

Furthermore, for many stability problems the corresponding base states are not constant and the normal modes analysis fails for that reason. To still get information on the dominant wavelength of the instability, amplifications of the initial condition can be computed numerically by solving the linearized system directly, i.e., as an initial value problem, in some cases combined with an optimal control approach to find the perturbation that leads to the largest amplification and therefore dominates the features of the instability $[8,10,40]$. This essentially takes up the idea of transient growth for nonnormal operators; see also [37]. Although, in principle, it is possible to numerically proceed in this way, it is desirable to gain the type of insight into the mathematical structure of the instability that is usually provided by spectral analysis for time-independent base states.

In the literature, a frequently used approach for time-dependent stability problems is the quasi-stationary, or so-called "frozen-mode," approximation where the time dependence appearing in the coefficients of the linearized system is treated as a parameter. For each value of this parameter, the eigenvalues are inspected with respect to their position in the complex plane. This has been described in a series of articles for a receding free film problem $[1,26]$ and for the evaporating solutal Marangoni instability [28]. Difficulties arise if the system changes significantly during the evolution of the perturbation, for example, if the frozen-mode analysis has unstable modes at the beginning which become stable later on. More generally, the problem with this approach is that it completely ignores the influence of the changing base state on the history of the perturbation.

An alternative to this quasi-stationary approach is provided if the base state changes slowly compared to the time-scale of the linearized system. Then a multiplescale method can be used to incorporate the change into an approximation of the evolution of the perturbation. In this direction a recent paper [15] develops an extension of the multiple-scale method for a parabolic convection-diffusion equation with slowly and nonperiodically changing coefficients and a slowly moving boundary that has been derived from a model for Bénard-Marangoni convection in a liquid layer of a volatile solvent and a nonvolatile polymer. There, the connection between the eigenvalues of the momentary system and the amplification of an initial perturbation was derived via a multiple-scale method making use of the time-scale separation between the fast diffusive time-scale which is relevant for the perturbation and the slow change of the concentration and thickness of the layer in the base state. The derivation included higher corrections in order to assess the validity of the asymptotic expansion. Alternatively, an asymptotic approximation via a WKB ansatz has been given in [14], and for the case of Rayleigh-Bénard convection with time-dependent heating a similar approach was investigated in [27].

In this study we focus on the linear stability analysis for the family of thin film 
problems characterized by degenerate or nondegenerate parabolic equations

$$
h_{t}+\nabla \cdot\left(h^{n} \nabla \Delta h\right)=0,
$$

with $0 \leq n<3$, where we present detailed analytical and numerical results for the cases $n=0$ and $n=2$, or $3 / 2<n<3$, respectively, wherever possible. In general this class of problems arises from mass conserving free boundary problems for the thin film height $h$. When an extended film is deposited onto a partially wettable substrate and then retracts after an initial rupture event has created a hole, the growing rim of the hole develops undulations along its spanwise direction $[4,5,11,36]$. The value of $n$ here depends on the condition imposed at the liquid-solid substrate.

It is well known that modeling the contact line is a very difficult topic. For $n=3$, for example, stress singularities near the contact line have to be taken into account due to the no-slip boundary condition. In [39] this difficulty is resolved via the inclusion of a thin film, referred to as precursor, which is much smaller than the typical height of the film. This technique also applies to other values of $n$, where the precursor film commonly represents the characteristic microscopic physics in a particular experiment $[2,23,35]$.

For large slip, which arises at the liquid-solid boundary as a manifestation of the non-Newtonian flow properties in shearing polymer films, the value is $n=2$ [35]. For this value of $n$, and in fact for all nonnegative $n<3$, imposing a fixed contact angle and a no-flux condition at $h=0$ leads to a problem formulation that is consistent with a moving contact line $[12,22]$. For $n=2$, undulations evolve into a characteristic finger-like pattern [33].

Rims of retracting solid films are susceptible to a similar instability which occurs in various practical applications. It can, for instance, be observed when thin silicon films are annealed and lead to the formation of fingering instabilities in the $\langle 100\rangle$ oriented front [25]. The fingers break down and form nanoislands at regular intervals, a process that is used in the fabrication of advanced nanodevices [18,24]. Formation of protrusions and pinch-off are also observed in experiments with gold films [19]. For interfaces of solids subject to surface diffusion [7,20,31,32,41], the material flux arises from gradients of the curvature-dependent chemical potential, leading to a fourth order, nonlinear equation for the evolution of the solid surface. Despite the different transport mechanism - surface diffusion [32] - the mathematical model governing the evolution of the height $h$ again leads to a differential equation of form (1.1) but with $n=0$. At the contact line, a fixed contact angle and no flux are commonly imposed as boundary conditions.

Since the thickness of the flat film into which the rim moves is assumed to be constant, while the rim itself grows, the time dependence cannot be removed by a single choice of self-similar coordinates. Thus, the linearization leads to a problem with time-dependent coefficients which has been approached by different authors. In [20], the linear stability of a rim in a solid film retracting under surface diffusion is addressed by a "frozen-mode" analysis, where the time dependence of the coefficients in the linearized problems is treated as a parameter.

Another approach was pursued in [33] for liquid dewetting, where the initial value problem resulting from the linearization was solved numerically to track the amplification of a perturbation. On the other hand, for long times, the leading order outer problem for the rim admits a traveling wave solution [12,23], since the inflation of the rim is slow once it has become large compared to the unperturbed film. If this growth is neglected, the traveling wave solution can be treated as stationary in 
a suitably chosen comoving frame of reference, and a normal-mode ansatz is possible again. Using scaling arguments to take into account the evolving base state, the amplification of a perturbation was inferred from the resulting spectrum [23,34]. A related approach was followed for a model of anisotropic solid dewetting in [9].

In this study we will develop a systematic WKB analysis to determine the evolution of a perturbation which appears at a moving contact line. The presented method applies to the class of thin film equations (1.1), where $0 \leq n<3$, commonly known to model thin films with large slip lengths or solid films where surface diffusion is dominant. For these problems, our approximation remains valid on the long time scale of the changing system and is used to derive in particular the wavenumber of the most amplified perturbation. This wavenumber is different from the most unstable one in a frozen-mode approach and is rapidly attained once the base state has entered an approximately self-similar scaling. Moreover, the asymptotic solution for the dispersion relation in the long wave limit reveals that the dependence of the growth rate on the wavenumber is linear, whereas in many other capillary instability problems it is quadratic.

The paper is organized as follows. After formulating the free boundary problem for (1.1) in section 2, we introduce the long time asymptotics for the base state in section 3. Detailed analytical and numerical results, referring to this and the following sections, are presented for the cases $n=0$ and $n=2$, or $3 / 2<n<3$, respectively, wherever possible. In section 4 , a WKB approximation for the associated linearized problem is developed and used to determine the most amplified mode and its wavenumber. Finally, we summarize our results in section 5 .

2. Formulation. We consider an evolution problem for the film profile $z=$ $h(x, y, t)$, given for $t>0$ by the $\operatorname{PDE}(1.1)$ on the time-dependent domain $\Omega=$ $\{(x, y) ; s(y, t)<x<\infty,-\infty<y<\infty\}$ and by appropriate conditions at the free boundary and in the far field, namely,

$$
\begin{aligned}
h & =0, & & x=s(y, t), \\
\nabla h \cdot \mathbf{n}_{s} & =\theta, & & x=s(y, t), \\
h^{n}\left(\nabla \Delta h \cdot \mathbf{n}_{s}\right) & =0, & & x=s(y, t), \\
\lim _{x \rightarrow \infty} h & =1 . & &
\end{aligned}
$$

The first three equations represent, respectively, the presence of a contact line with fixed slope $\theta$ and no flux at $x=s(y, t)$, and the last condition prescribes that we have a flat film at $x \rightarrow \infty$, with a thickness that has been scaled to one. Here,

$$
\mathbf{n}_{s}=\left(1,-\partial_{y} s\right) /\left(1+\left(\partial_{y} s\right)^{2}\right)^{1 / 2}
$$

is the normalized normal vector along $x=s(y, t)$ in the $(x, y)$-plane pointing into $\Omega$, as sketched in Figure 1. The initial conditions

$$
h(x, y, 0)=h_{i}(x, y), \quad s(y, 0)=s_{i}(y)
$$

are assumed to be chosen to be consistent with (2.1). We note that the interval $0 \leq n<3$ subdivides into essentially three separate cases, $n=0,0<n \leq 3 / 2$, and $3 / 2<n<3$. The first is instructive because the PDE is linear, and therefore the analytic results can be carried out much further. While it has a simple asymptotic structure, the rim profile pinches off after a finite time. While for $n>3 / 2$ this does 


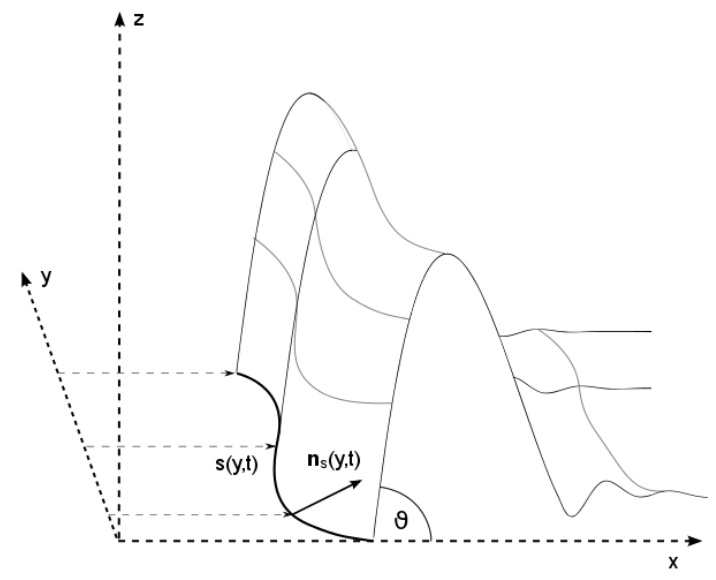

FIG. 1. A sketch of a retracting rim with a sinusoidal perturbation in the spanwise (y-) direction.

not happen, the film profile decomposes into different asymptotic scaling regimes that need to be matched. The intermediate case combines both features and is not pursued here further since it does not add to the discussion of the stability analysis.

3. Base state. We consider a time-dependent base state $h_{b}=h_{b}(x, t)$ which is constant in the $y$-direction and has a straight contact line $s_{b}=s_{b}(t)$. We transform to a coordinate system moving with the contact line via $h_{b}(x, t)=\tilde{h}_{b}(\tilde{x}, t), \tilde{x}=x-s_{b}(t)$, so that after dropping the tilde, we have

$$
\begin{aligned}
\partial_{t} h_{b}-\dot{s}_{b} \partial_{x} h_{b}+\partial_{x}\left(h_{b}^{n} \partial_{x x x} h_{b}\right) & =0 & \text { for } x \geq 0, \\
h_{b}=0, \quad \partial_{x} h_{b}=\theta, \quad h_{b}^{n} \partial_{x x x} h_{b} & =0 & \text { at } x=0, \\
\lim _{x \rightarrow \infty} h_{b} & =1 . &
\end{aligned}
$$

Notice that due to mass conservations, $h_{b}$ satisfies

$$
\int_{0}^{\infty} h_{b}(x, t)-h_{b}(x, 0) d x=s_{b}(t) .
$$

In fact, an equivalent formulation of (3.1) that we use as the basis for the numerical discretization is achieved by replacing the third condition in (3.1b) by (3.2). The domain is truncated at $x=L_{\infty}$, where $L_{\infty}$ is chosen large enough so that the growing rim structure is accommodated up to the desired time, typically much larger than 100. The resulting system is discretized in space using standard centered finite differences on an equidistant grid (with a typical grid spacing $\Delta x=0.1$ ) and a trapezoidal rule for (3.2), and in time with a (fully implicit) Euler scheme. The latter is combined with step doubling and extrapolation for higher accuracy and time step control. For the initial conditions, we used a smoothed Heaviside profile

$$
s_{b}(0)=0, \quad h_{b}(x, 0)= \begin{cases}1-\theta(x-1)^{2} / 2 & \text { for } 0 \leq x \leq 1, \\ 1 & \text { for } x \geq 1 .\end{cases}
$$

Copyright (c) by SIAM. Unauthorized reproduction of this article is prohibited. 

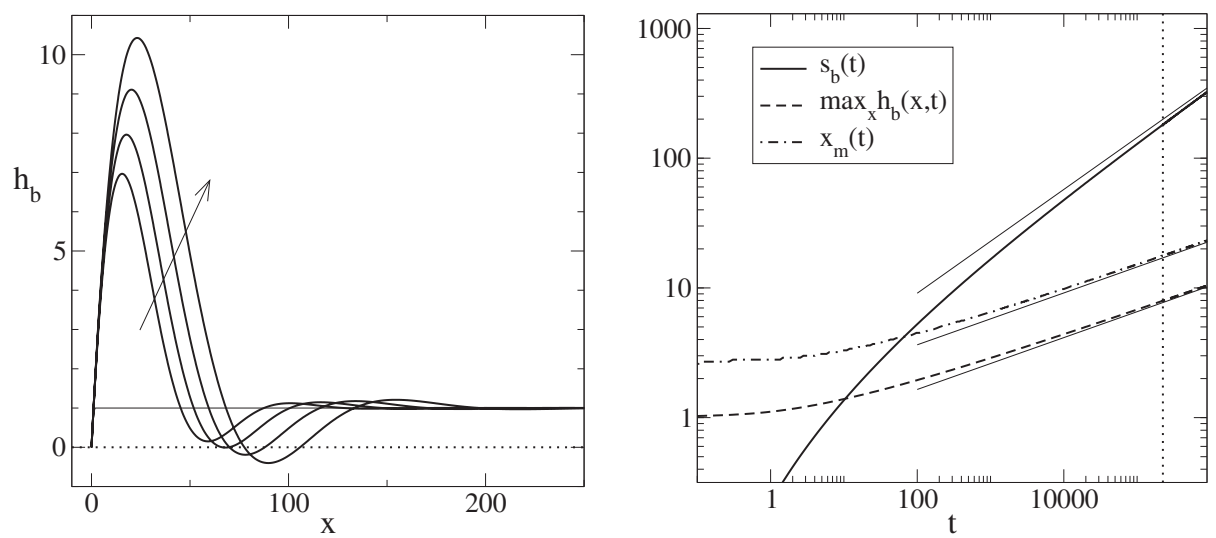

Fig. 2. Left: Profiles of the base state for $\theta=1$ at different times, obtained by solving (3.1) numerically (using (3.2) instead of the third boundary condition in (3.1b)) for $\theta=1$ and $n=0$. The initial data (3.3) is shown by a thin solid line, and later times are shown by thicker solid lines, for $t=t_{r} / 2, t=t_{r}, 2 t_{r}, 4 t_{r}$, where $t_{r} \equiv 2.25 \times 10^{5}$ is the time at which the first minimum of the profile to the right of the rim hits zero. All profiles are shown in a comoving frame of reference with the contact line fixed at the origin. Right: Evolution of the contact line $s_{b}$, the maximum value $\max _{x} h$, and the position $x_{m}$ where the maximum is achieved. The thin straight lines are the asymptotic results (3.16). The thin dotted vertical line indicates the rupture time $t=t_{r}$.
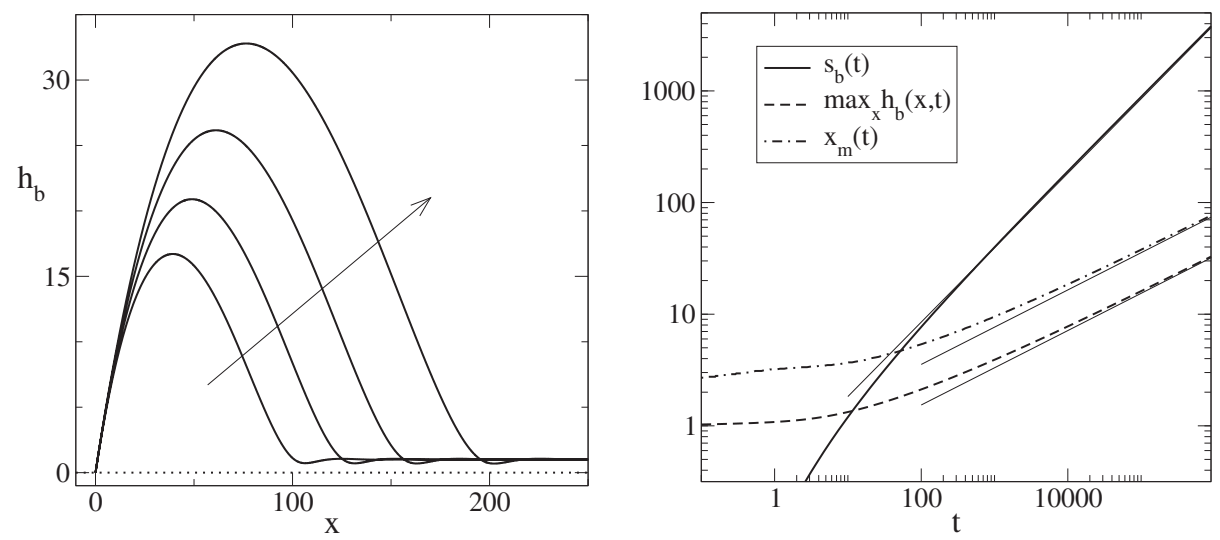

FIG. 3. Left: Profiles of the base state for $\theta=1$ at different times, obtained by solving (3.1) numerically (using (3.2) instead of the third boundary condition in (3.1b)) for $\theta=1$ and $n=2$. The initial data (3.3) is shown by a thin solid line, and later times are shown by thicker solid lines, for $t=t_{\text {ref }} / 2, t=t_{\text {ref }}, 2 t_{\text {ref }}, 4 t_{\text {ref }}$, where $t_{r e f} \equiv 2.25 \times 10^{5}$. All profiles are shown in a comoving frame of reference with the contact line fixed at the origin. Right: Evolution of the contact line $s_{b}$, the maximum value $\max _{x} h$, and the position $x_{m}$ where the maximum is achieved. The thin straight lines are the asymptotic results (3.24a), (3.24b), (3.24c), respectively.

Long time solution. Consistent with the analysis in $[12,22,31,35]$, the numerical solutions show that for long times, $t \rightarrow \infty$, the position of the contact line $s_{b}$ and the maximum height of the rim evolve according to a power law; See Figures 2(right) and 3 (right). We briefly summarize and adapt the previous results to the case studied here and compare it with the numerical results.

To capture the long time behavior analytically, we introduce a second time-scale $\tau=\delta t$, where $\delta \ll 1$, and consider the limit $\delta \rightarrow 0$ with $\tau$ fixed. The observed power 
law behavior suggests rescaling $s_{b}$ by $\delta^{-\sigma}$, where the exponent $\sigma>0$ needs to be determined. Mass conservation (3.2) and the assumption that height and width of the rim rescale identically then motivates the choices

$$
s_{b}(t)=\delta^{-\sigma} S_{b}(\tau), \quad x=\delta^{-\sigma / 2} X, \quad h_{b}=\delta^{-\sigma / 2} H_{b} .
$$

Inserting these scalings into (3.1a) reveals that only the second and third terms can be balanced (the scaling of the term with the time derivative always being much smaller than the other two), and this balance implies $\sigma=2 /(5-n)$. Thus, the rescaled equations read

$$
\begin{aligned}
\delta^{1 /(5-n)} \partial_{\tau} H_{b}-\dot{S}_{b} \partial_{X} H_{b}+\partial_{X}\left(H_{b}^{n} \partial_{X X X} H_{b}\right) & =0 \quad \text { for } X \geq 0 \\
H_{b}=0, \quad \partial_{X} H_{b}=\theta, \quad H_{b}^{n} \partial_{X X X} H_{b} & =0 \quad \text { at } X=0, \\
\lim _{X \rightarrow \infty} H_{b} & =\delta^{1 /(5-n)}
\end{aligned}
$$

where we remark that the dot over $S_{b}$ now denotes derivatives with respect to $\tau$. Next, we expand

$$
H_{b}(X, \tau ; \delta)=H_{b, 0}(X, \tau)+O\left(\delta^{1 /(5-n)}\right), \quad S_{b}(\tau ; \delta)=S_{b, 0}(\tau)+O\left(\delta^{1 /(5-n)}\right) .
$$

The leading order problem is given by $(3.5 \mathrm{a})$ and (3.5b) after dropping the $\delta^{1 /(5-n)}$ term that appears in the ODE. Integrating and using the leading order boundary conditions

$$
H_{b, 0}=0, \quad \partial_{X} H_{b}=\theta, \quad H_{b, 0}^{n} \partial_{X X X} H_{b, 0}=0 \quad \text { at } X=0
$$

then yields

$$
H_{b, 0}^{n-1} \partial_{X X X} H_{b, 0}=\dot{S}_{b, 0} .
$$

Notice that $\theta$ and $\dot{S}_{b, 0}$ can be removed from (3.7) and (3.8) by rescaling:

$$
X=\theta^{n /(3-n)} \frac{\xi}{\dot{S}_{b, 0}^{1 /(3-n)}}, \quad H_{b, 0}=\theta^{3 /(3-n)} \frac{\phi_{b}}{\dot{S}_{b, 0}^{1 /(3-n)}} .
$$

Case $\boldsymbol{n}=\mathbf{0}$. In this case, we simply use the leading order version of the condition (3.5c) in the far field,

$$
\lim _{X \rightarrow \infty} H_{b, 0}=0 .
$$

The boundary value problem (3.7), (3.8), (3.10) is linear and has the solution

$$
H_{b, 0}(X, \tau)=\frac{2}{\sqrt{3}} \frac{\theta}{\dot{S}_{b, 0}^{1 / 3}} \exp \left(-\frac{\dot{S}_{b, 0}^{1 / 3} X}{2}\right) \sin \left(\frac{\sqrt{3}}{2} \dot{S}_{b, 0}^{1 / 3} X\right) .
$$

Moreover, to leading order, we obtain from (3.2)

$$
\int_{0}^{\infty} H_{b, 0} d x=S_{b, 0} .
$$

Inserting the solution for $H_{b, 0}$ yields a differential equation for $S_{b, 0}$,

$$
S_{b, 0}^{3} \dot{S}_{b, 0}^{2}=M \theta^{3},
$$

Copyright $@$ by SIAM. Unauthorized reproduction of this article is prohibited. 
where

$$
M \equiv\left(\int_{0}^{\infty} \phi_{b} d \xi\right)^{3}
$$

and assuming that $S_{b, 0}(0)=0$ gives the solution

$$
S_{b, 0}=(5 / 2)^{2 / 5} \theta^{3 / 5} \tau^{2 / 5} .
$$

Written in the original variables, we have for large $t$

$$
s_{b} \sim(5 / 2)^{2 / 5} \theta^{3 / 5} t^{2 / 5}=1.44 \theta^{3 / 5} t^{2 / 5} .
$$

Furthermore, for the maximum of the base state $\max _{x} h_{b}$ and the value of $x=x_{m}(t)$ where it is achieved, we obtain

$$
\begin{aligned}
\max _{x} h_{b}(x, t) & \sim(2 / 5)^{-1 / 5} \mathrm{e}^{-\pi / \sqrt{27}} \theta^{4 / 5} \mathrm{t}^{1 / 5}=0.656 \theta^{4 / 5} \mathrm{t}^{1 / 5} \\
x_{m}(t) & \sim \frac{\pi}{\sqrt{27}} 2^{4 / 5} 5^{1 / 5} \theta^{-1 / 5} t^{1 / 5}=1.45 \theta^{-1 / 5} t^{1 / 5}
\end{aligned}
$$

The numerical profiles for $H_{b}$ are shown in Figure 2(left). The film quickly forms a capillary rim that grows for large times in an approximately self-similar fashion, except in the far field, which is constant, with approximately the same scaling factor for the height and the width so it maintains a fixed contact angle. Notice that at some stage, the first local minimum to the right of the rim touches zero. For the simulations shown here, with $\theta=1$, this happens around $t=t_{r} \equiv 2.25 \times 10^{5}$, with $\max _{h}=7.96$. Physically, this means that the diffusing film ruptures and sheds material that collects into an equilibrium shape, while the dewetting by surface diffusion resumes with a new contact line. Nevertheless, the mathematical formulation does allow for solutions that are negative, and in order to understand the long time asymptotic structure, it is convenient to investigate these solutions beyond $t_{r}$. Since the rupture occurs for large $t$, we can expect the asymptotic solutions to yield reasonable approximations even for a range of $t<t_{r}$. Indeed, all three asymptotic approximations (3.16a), (3.16b), $(3.16 \mathrm{c})$ agree well with the numerical results for sufficiently large $t$, as can be seen in Figure 2(right).

Case $\boldsymbol{n}>\mathbf{3} / \mathbf{2}$. Note that in this case, the rim decomposes into different scalings, where the rim takes on the role of the outer solution and needs to be matched to further layers on the right. The details of this matching depend on $n$ and are quite intricate in general [22]. For $n=2$, there is just one inner layer, and the matching approach for this particular value of $n$ has been presented in [12,35]. However, the procedure can be generalized to $3 / 2<n<3$, which is carried out in Appendix A, and here we will give only the matching condition. According to the similar matching behavior, the entire section refers to the cases where $3 / 2<n<3$.

In outer coordinates, we have the matching condition

$$
H_{b, 0}(X, \tau) \sim\left(\frac{n^{3}}{3(3-n)(2 n-3)}\right)^{1 / n} \dot{S}_{b, 0}^{1 / n}\left(W_{b, 0}-X\right)^{3 / n} \quad \text { for } X \rightarrow W_{b, 0}
$$

where $W_{b, 0}=W_{b, 0}(\tau)>0$ denotes the leading order expression for the matching point in between the outer rim and the undisturbed film on the right-hand side (see Appendix A for a detailed derivation).

Copyright (c) by SIAM. Unauthorized reproduction of this article is prohibited. 
To leading order conservation of mass (3.2) yields

$$
\int_{0}^{W_{b, 0}(\tau)} H_{b, 0} d x=S_{b, 0}
$$

Inserting the scalings (3.9), we obtain

$$
\begin{array}{ll}
\phi_{b, 0}^{n-1} \partial_{\xi \xi \xi} \phi_{b, 0}=1 & \text { for } 0 \leq \xi<d_{b}, \\
\phi_{b, 0}=0, \quad \partial_{\xi} \phi_{b, 0}=1, \quad \phi_{b, 0}^{n} \partial_{\xi \xi \xi} \phi_{b, 0}=0 & \text { at } \xi=0, \\
\phi_{b, 0} \sim\left(\frac{n^{3}}{3(3-n)(2 n-3)}\right)^{1 / n}\left(d_{b}-\xi\right)^{3 / n} & \text { for } \xi \rightarrow d_{b},
\end{array}
$$

where $d_{b}$ arises from rescaling $W_{b, 0}(\tau)$,

$$
W_{b, 0}(\tau)=\theta^{n /(3-n)} \frac{d_{b}}{\dot{S}_{b, 0}^{1 /(3-n)}} .
$$

Moreover, we obtain from (3.18) the differential equation

$$
S_{b, 0}^{(3-n)} \dot{S}_{b, 0}^{2}=M \theta^{(3+n)},
$$

where $M$ is given by

$$
M=\left(\int_{0}^{d_{b}} \phi_{b, 0} d \xi\right)^{3-n} .
$$

The differential equation (3.21) for $\dot{S}_{b, 0}$ with $S_{b, 0}(0)=0$ has the solution

$$
S_{b, 0}=\left(\frac{5-n}{2}\right)^{2 /(5-n)} M^{1 /(5-n)} \theta^{(3+n) /(5-n)} \tau^{2 /(5-n)} .
$$

In general, (3.19) has to be solved numerically. For $n=2$, the order can be reduced by one integration, and this gives $d_{b}=1 / 2$ in the process [12,35]; numerically, one finds $M=2.72 \times 10^{-2}$ (see [35]), and from this,

$$
s_{b} \sim 0.394 \theta^{5 / 3} t^{2 / 3}
$$

for large $t$, where we have converted back to the original variables.

Moreover, for the maximum of the base state $\max _{x} h_{b}$ and the value of $x=x_{m}(t)$ where it is achieved, we obtain

$$
\begin{aligned}
\max _{x} h_{b}(x, t) & \sim(2 / 3)^{-1 / 3} M^{-1 / 3} \max _{\xi} \phi_{b} \theta^{4 / 3} t^{1 / 3}=0.331 \theta^{4 / 3} t^{1 / 3}, \\
x_{m}(t) & \sim(2 / 3)^{-1 / 3} M^{-1 / 3} \xi_{m} \theta^{1 / 3} t^{1 / 3}=0.765 \theta^{1 / 3} t^{1 / 3},
\end{aligned}
$$

where $\max _{\xi} \phi_{b}=0.870 \times 10^{-1}$ and $\xi_{m}=0.201$ were obtained from the numerical solution for (3.19) computed in [35]. The agreement between asymptotical and numerical results is excellent. Notice that the first dip following the maximum in the profiles remains shallow even as the rims grow, thus showing no indication of a rupture. This is consistent with the prediction that rupture does not occur for $n>3 / 2$; see [12,22].

Copyright (c) by SIAM. Unauthorized reproduction of this article is prohibited. 


\section{Linear stability.}

4.1. Formulation. We first shift the full problem (1.1) into the reference frame moving with the contact line of the base state $s_{b}(t)$ by using the same change of variables as that for the base state equation, that is, $h(x, y, t)=\tilde{h}(\tilde{x}, \tilde{y}, t), \tilde{x}=x-s_{b}(t)$, and $y=\tilde{y}$. After dropping the tildes, the PDE becomes

$$
\partial_{t} h-\dot{s}_{b} \partial_{x} h+\nabla \cdot\left(h^{n} \nabla \Delta h\right)=0,
$$

while the boundary and far field conditions remain unchanged. We introduce perturbations of the base state $h_{b}$ and $s_{b}$ of the form

$$
h(x, y, t)=h_{b}(x, t)+\varepsilon h_{1}(x, y, t), \quad s(y, t)=\varepsilon s_{1}(y, t),
$$

with $0<\varepsilon \ll 1$ and Fourier transform,

$$
h_{1}(x, y, t)=\int_{-\infty}^{\infty} \hat{h}_{1}(x, t ; q) \exp (i q y) d q, \quad s_{1}(y, t)=\int_{-\infty}^{\infty} \hat{s}_{1}(t ; q) \exp (i q y) d q .
$$

In this way we obtain, to $O(\varepsilon)$,

$$
\begin{aligned}
\partial_{t} \hat{h}_{1}-\hat{\mathcal{L}} \hat{h}_{1} & =0 & & \text { for } x \geq 0, \\
\hat{h}_{1}=-\theta \hat{s}_{1}, \quad \partial_{x} \hat{h}_{1} & =-\hat{s}_{1} \partial_{x x} h_{b} & & \text { at } x=0, \\
h_{b}^{n}\left(\partial_{x x x} \hat{h}_{1}-q^{2} \partial_{x} \hat{h}_{1}\right)+n h_{b}^{n-1} \partial_{x x x} h_{b} \hat{h}_{1}-\dot{s}_{b} \hat{h}_{1} & =0 & & \text { at } x=0, \\
\lim _{x \rightarrow \infty} \hat{h}_{1} & =0, & &
\end{aligned}
$$

where

$\hat{\mathcal{L}} \hat{h}_{1} \equiv \dot{s}_{b} \partial_{x} \hat{h}_{1}-\partial_{x}\left[h_{b}^{n}\left(\partial_{x x x} \hat{h}_{1}-q^{2} \partial_{x} \hat{h}_{1}\right)+n\left(\partial_{x x x} h_{b}\right) h_{b}^{n-1} \hat{h}_{1}\right]+q^{2} h_{b}^{n}\left(\partial_{x x} \hat{h}_{1}-q^{2} \hat{h}_{1}\right)$.

For each value of $q$, we need to provide an initial perturbation of the Fourier mode with wavenumber $q$ via

$$
\hat{h}_{1}\left(x, t_{0} ; q\right)=\hat{h}_{1, t_{0}}(x ; q), \quad \hat{s}_{1}\left(t_{0} ; q\right)=-\hat{h}_{1, t_{0}}(0 ; q) / \theta,
$$

where, for simplicity, we have chosen the perturbation of the contact line to be consistent with the first boundary condition in $(4.3 \mathrm{~b})$.

For $n=0$, the problem (4.3) was solved with an extension of the numerical scheme used for the base state problem (3.1), that is, finite differences in space and an extrapolated implicit Euler scheme in time. The initial perturbation $\hat{h}_{1, t_{0}}$ was set equal to the derivative $\partial_{x} h_{b}(x, 0)$ of the initial condition for the base state (3.3). A different approach was used for $n=2$, as explained in the part of subsection 4.3 focusing on this case. In both cases, the codes were constructed to track the evolution of $\hat{h}_{1}$ for several wavenumbers $q$ simultaneously.

4.2. Asymptotic analysis. Notice that the above problem remains coupled to the base state via the second term in (4.3a) and coefficients in the right-hand sides of the second and third boundary conditions in $(4.3 \mathrm{~b})$, and that the base state is time dependent. The problem can therefore not be approached by normal modes/separation 
of variables. For time-independent base states, separation of variables leads to an eigenvalue problem, and the long time evolution of the perturbation is usually dictated by the top eigenvalue, which is often discrete in typical capillary instabilities. We can, however, exploit the fact that for longer times, the base state evolves on a slower time-scale than the typical perturbations and approaches an asymptotically self-similar form. The evolution of the perturbations can then be recovered from the eigenvalue information by using a multiple-scale method or WKB approach $[15,27]$. We therefore introduce again the slow time-scale $\tau$ via $t=\tau / \delta$, together with the scalings in (3.4) and corresponding scalings for the perturbation variables and for the wavenumber, that is,

$$
\hat{h}_{1}=\delta^{-1 /(5-n)} \Phi, \quad \hat{s}_{1}=\delta^{-1 /(5-n)} \hat{S}, \quad q=\delta^{1 /(5-n)} Q,
$$

such that the stability problem transforms into

$$
\begin{array}{rlrl}
\delta^{1 /(5-n)} \partial_{\tau} \Phi-\mathcal{L}(\tau) \Phi & =0 & & \text { for } X \geq 0, \\
\theta \partial_{X} \Phi-\Phi \partial_{X X} H_{b}=0 & \text { at } X=0, \\
H_{b}^{n}\left(\partial_{X X X} \Phi-Q^{2} \partial_{X} \Phi\right)+n H_{b}^{n-1} \partial_{X X X} H_{b} \Phi-\dot{S}_{b} \Phi=0 & \text { at } X=0, \\
\lim _{X \rightarrow \infty} \Phi=0 . &
\end{array}
$$

Here

$$
\begin{gathered}
\mathcal{L}(\tau) \Phi \equiv \dot{S}_{b} \partial_{X} \Phi-\partial_{X}\left[H_{b}^{n}\left(\partial_{X X X} \Phi-Q^{2} \partial_{X} \Phi\right)+n\left(\partial_{X X X} H_{b}\right) H_{b}^{n-1} \Phi\right] \\
+Q^{2} H_{b}^{n}\left(\partial_{X X} \Phi-Q^{2} \Phi\right)
\end{gathered}
$$

and we combined the two boundary conditions in (4.3b) into one in (4.5b).

To exploit the fact that the coefficients in this linear stability problem only change slowly in time, we make a WKB ansatz by introducing

$$
\Phi(X, \tau)=\Psi(X, \tau) \exp \left(\frac{\sigma(\tau)}{\delta^{1 /(5-n)}}\right)
$$

giving

$$
\begin{aligned}
\delta^{1 /(5-n)} \partial_{\tau} \Psi+\Psi \partial_{\tau} \sigma & =\mathcal{L}(\tau) \Psi & & \text { for } X \geq 0, \\
\theta \partial_{X} \Psi-\Psi \partial_{X X} H_{b} & =0 & & \text { at } X=0, \\
H_{b}^{n}\left(\partial_{X X X} \Psi-Q^{2} \partial_{X} \Psi\right)+n H_{b}^{n-1} \partial_{X X X} H_{b} \Psi-\dot{S}_{b} \Psi & =0 & & \text { at } X=0, \\
\lim _{X \rightarrow \infty} \Psi & =0, & &
\end{aligned}
$$

and then applying the asymptotic expansions

$$
\Psi(X, \tau ; \delta)=\Psi_{0}(X, \tau)+O\left(\delta^{1 /(5-n)}\right), \quad \sigma(\tau ; \delta)=\sigma_{0}(\tau)+O\left(\delta^{1 /(5-n)}\right),
$$

together with the expansions (3.6) for the base state on which the coefficients of this system depend.

Exploiting (3.8), the leading order problem is

$$
\begin{aligned}
& \lambda(\tau) \Psi_{0}=\mathcal{L}_{0}(\tau) \Psi_{0} \quad \text { for } X \geq 0, \\
& \theta \partial_{X} \Psi_{0}-\Psi_{0} \partial_{X X} H_{b, 0}=0 \quad \text { at } X=0, \\
& H_{b, 0}^{n}\left(\partial_{X X X} \Psi_{0}-Q^{2} \partial_{X} \Psi_{0}\right)-(1-n) \dot{S}_{b, 0} \Psi_{0}=0 \quad \text { at } X=0 \text {, }
\end{aligned}
$$

Copyright (c) by SIAM. Unauthorized reproduction of this article is prohibited. 
where

$$
\begin{aligned}
& \lambda(\tau) \equiv \partial_{\tau} \sigma_{0} \\
& \mathcal{L}_{0}(\tau) \Psi_{0} \equiv(1-n) \dot{S}_{b, 0} \partial_{X} \Psi_{0}-\partial_{X}\left[H_{b, 0}^{n}\left(\partial_{X X X} \Psi_{0}-Q^{2} \partial_{X} \Psi_{0}\right)\right] \\
& \quad+Q^{2} H_{b, 0}^{n}\left(\partial_{X X} \Psi_{0}-Q^{2} \Psi_{0}\right) .
\end{aligned}
$$

For $n=0$, the leading order of (4.8d) provides a condition in the far field, while for $n=2$, the limit $\delta \rightarrow 0$ is singular and matching conditions arise at $X=W_{b, 0}(\tau)$ instead. The latter will be discussed in detail further below and in Appendix A. Our main attention is directed towards determining the perturbations with largest amplification, and therefore towards the eigenvalue with largest real part, or top eigenvalue.

Rescaling the variables as in (3.9), and in (3.20) for $n>0$, and introducing appropriate scales for the single-mode perturbation, wavenumber, and eigenvalue,

$$
\Psi_{0}=\theta^{3 /(3-n)} \frac{\phi_{1}}{\dot{S}_{b, 0}^{1 /(3-n)}}, \quad Q=\dot{S}_{b, 0}^{1 /(3-n)} \frac{\tilde{Q}}{\theta^{n /(3-n)}}, \quad \lambda=\dot{S}_{b, 0}^{(4-n) /(3-n)} \frac{\tilde{\lambda}}{\theta^{n /(3-n)}},
$$

leads to the parameter-free eigenvalue problem

$$
\begin{aligned}
\tilde{\lambda} \phi_{1} & =\tilde{\mathcal{L}} \phi_{1} & \text { for } \xi & \geq 0, \\
\partial_{\xi} \phi_{1}-\phi_{1} \partial_{\xi \xi} \phi_{b} & =0 & \text { at } \xi & =0, \\
\phi_{b}^{n}\left(\partial_{\xi \xi \xi} \phi_{1}-\tilde{Q}^{2} \partial_{\xi} \phi_{1}\right)-(1-n) \phi_{1} & =0 & \text { at } \xi & =0,
\end{aligned}
$$

where

$$
\tilde{\mathcal{L}} \phi_{1} \equiv(1-n) \partial_{\xi} \phi_{1}-\partial_{\xi}\left[\phi_{b}^{n}\left(\partial_{\xi \xi \xi} \phi_{1}-\tilde{Q}^{2} \partial_{\xi} \phi_{1}\right)\right]+\tilde{Q}^{2} \phi_{b}^{n}\left(\partial_{\xi \xi} \phi_{1}-\tilde{Q}^{2} \phi_{1}\right)
$$

plus the remaining far field or matching condition.

Case $\boldsymbol{n}=\mathbf{0}$. For $n=0$, the remaining condition is

$$
\lim _{\xi \rightarrow \infty} \phi(\xi)=0
$$

Moreover, recalling (3.11), we have

$$
\phi_{b}(\xi)=\frac{2}{\sqrt{3}} \exp \left(-\frac{\xi}{2}\right) \sin \left(\frac{\sqrt{3}}{2} \xi\right)
$$

and thus we can set

$$
\left.\partial_{\xi \xi} \phi_{b}\right|_{\xi=0}=-1
$$

in (4.14b). Notice that this is the only spot in (4.14) where the solution of the base state is needed, in contrast to the situation for $n>0$.

Copyright $@$ by SIAM. Unauthorized reproduction of this article is prohibited. 
Case $n>\mathbf{3} / \mathbf{2}$. For $n=2$ we have a second contact line near the undisturbed film and hence need to determine a matching condition for the inner region ahead of the hump. Similarly as for the base state (see section 3) the matching procedure can be generalized, and consequently this entire section refers to the cases where $3 / 2<n<3$.

In outer coordinates we have the behavior

$$
\Psi_{0}(X, \tau) \sim 3\left(\frac{n^{3-n}}{3(3-n)(2 n-3)}\right)^{1 / n} S_{b, 0}^{1 / n} W_{1,0}\left(W_{b, 0}(\tau)-X\right)^{(3-n) / n}
$$

for $X \rightarrow W_{b, 0}(\tau)$, where $W_{1,0}(\tau)$ represents the $\delta$-leading order perturbation of the second contact line. This matches with the inner expansion; for more details, see Appendix A. Applying the scales (3.9) and (4.13) then reveals

$$
\phi_{1} \sim 3\left(\frac{n^{3-n}}{3(3-n)(2 n-3)}\right)^{1 / n} d_{1}\left(d_{b}-\xi\right)^{(3-n) / n} \quad \text { for } \xi \rightarrow d_{b},
$$

where $d_{b}=\dot{S}_{b, 0}^{1 /(3-n)} /\left(\theta^{n /(3-n)}\right) W_{b, 0}$ and $d_{1}=\dot{S}_{b, 0}^{1 /(3-n)} /\left(\theta^{n /(3-n)}\right) W_{1,0}$, which completes the eigenvalue problem (4.14).

\section{Eigenvalue analysis.}

Case $\boldsymbol{n}=\mathbf{0}$. The general solution $\phi_{1}$ of the homogeneous linear differential equation (4.14a) has the form

$$
\phi_{1}(\xi)=\sum_{j=1}^{4} c_{j} \exp \left(k_{j} \xi\right),
$$

where the $k_{j}$ are the roots of the fourth order polynomial

$$
k_{j}^{4}-2 \tilde{Q}^{2} k_{j}^{2}-k_{j}+\tilde{Q}^{4}+\tilde{\lambda}=0 .
$$

We first restrict our situation to the case where the roots have negative real part, such that the corresponding contributions to (4.20) satisfy the boundary conditions (4.15) at $\xi \rightarrow \infty$. As shown in Appendix B, there are exactly two of these roots, say $k_{1}$ and $k_{2}$, and we note that these solutions arise for all $\tilde{Q} \geq 0$, in particular when the real part of $\tilde{\lambda}$ is positive, i.e., $\Re(\tilde{\lambda})>0$, which is the region of the complex plane associated with unstable modes.

Restricting our attention to these solutions, we have

$$
\phi_{1}(\xi)=c_{1} \exp \left(k_{1} \xi\right)+c_{2} \exp \left(k_{2} \xi\right) .
$$

Substituting (4.22) into (4.14b) and (4.14c) gives a homogeneous linear system of equations for the coefficients $c_{1}, c_{2}$, with the coefficient matrix

$$
A=\left(\begin{array}{cc}
k_{1}+1 & k_{2}+1 \\
k_{1}^{3}-\tilde{Q}^{2} k_{1}-1 & k_{2}^{3}-\tilde{Q}^{2} k_{2}-1
\end{array}\right),
$$

which depends on $\tilde{\lambda}$ via $k_{1}$ and $k_{2}$. The eigenvalues $\tilde{\lambda}$ are found by solving $\operatorname{det} A=0$. Numerically, this can be done by finding the intersection of the level sets $\Re(\operatorname{det} A)=0$ and $\Im(\operatorname{det} A)=0$ in the $(\Re(\tilde{\lambda}), \Im(\tilde{\lambda}))$-plane. It turns out that for the intersection with largest real part $\Re(\tilde{\lambda})$, the imaginary part $\Im(\tilde{\lambda})$ is zero (within the numerical 

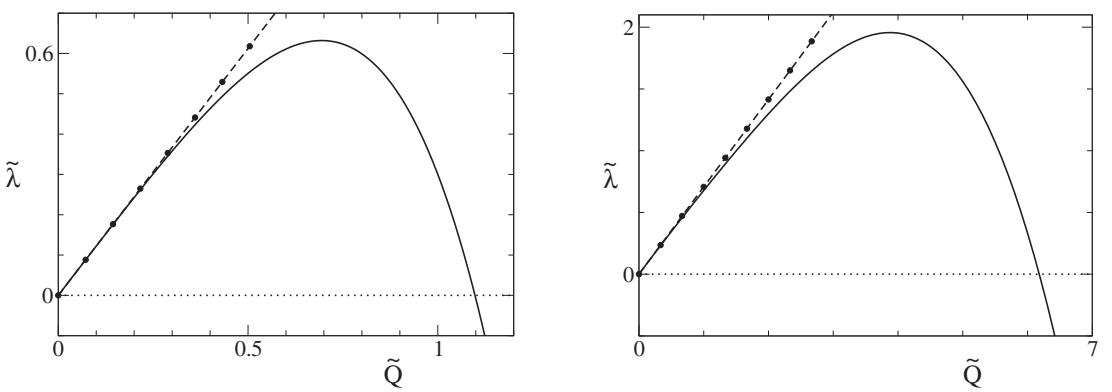

FIG. 4. Left: Top eigenvalue in the case $n=0$ for a range of wavenumbers. Results for the exact numerical results for (4.14) with (4.15) and (4.17) are shown by a solid line, and those for the long wave analysis (4.24) are shown by a dashed line with symbols. Right: Top eigenvalue in the case $n=2$, resulting from solving (4.14), (4.19). Line styles carry over from the left.

tolerance). Plotting this $\tilde{\lambda}$ as a function of $\tilde{Q}$ results in the dispersion relation shown in Figure 4(left). We also verified that for this $\tilde{\lambda}(\tilde{Q})$ and for all $\tilde{Q}>0$, exactly two roots of (4.21) have nonpositive real part, even when, for $\tilde{Q}>1.10$, the value of $\tilde{\lambda}(\tilde{Q})$ is negative. Thus, $\tilde{\lambda}$ is an isolated eigenvalue.

For $\tilde{\lambda}$ with sufficiently small real part, more than two roots of the characteristic polynomial can have negative real part. Then imposing (4.14b) and (4.14c) leads to an undetermined system for the coefficients of the decaying exponentials, i.e., a system which always has nontrivial solutions. Therefore, these $\tilde{\lambda}$ form the essential spectrum of the operator. Due to our previous remark, the essential spectrum is restricted, for all values of $\tilde{Q}$, to a subset of the complex plane with $\Re(\tilde{\lambda})<0$. For $\tilde{Q}=0$, the origin $\tilde{\lambda}=0$ is equal to the previously discussed isolated eigenvalue and also lies on the boundary of the essential spectrum, which is also the limit for $\tilde{Q} \rightarrow 0$ of the isolated eigenvalue discussed above. Similar situations are observed in the analysis of the stability of waves in conservation laws [3, 29, 30,42].

Notice that the dispersion relation appears to be approximately linear for $\tilde{Q}<$ 0.3 , despite the fact that only even powers of $\tilde{Q}$ appear in (4.14). In fact, for long wavelengths, the eigenvalues can be approximated in terms of small- $Q$ perturbations of the translational mode,

$$
\phi_{1}=\partial_{\eta} \phi_{b}+\phi_{1,1}|\tilde{Q}|+O\left(\tilde{Q}^{2}\right), \quad \tilde{\lambda}=\tilde{\lambda}_{1}|\tilde{Q}|+O\left(\tilde{Q}^{2}\right) .
$$

In addition to the typically expected even powers of $\tilde{Q}$, the expansion also contains odd powers of $\tilde{Q}$ which is due to the fact that the eigenvalue $\tilde{\lambda}=0$ at $\tilde{Q}=0$ is degenerate, i.e., belongs to a Jordan block. This is very similar to the situation found for dewetting a liquid in a slip-dominated case [23]. Proceeding as in that reference, we obtain the value $\tilde{\lambda}_{1}=(3 / 2)^{1 / 2}=1.22$; details are given in Appendix C. Good agreement with the numerically found eigenvalues up to $\tilde{Q}=0.3$ is seen in Figure 4(left) for the positive value for $\tilde{\lambda}_{1}$. The long wave analysis also allows for an expansion where the $O(\tilde{Q})$ term has the reverse sign, but it turns out that this leads to $\tilde{\lambda}$ in regions of the complex plane where the characteristic equation (4.21) has more than two roots with negative real part, i.e., to $\tilde{\lambda}$ which are in the essential spectrum.

The long wave analysis confirms that as $\tilde{Q} \rightarrow 0$, the top eigenvalue decreases to zero and thus converges to a point on the boundary of the essential spectrum. Since the expansions for isolated eigenvalues (see, for example, Hennessy and Münch [15]) may lose validity if two eigenvalues approach each other, we expect that a similar loss 
of validity could occur here. This means a separate analysis is needed for the case where $\tilde{Q}$ is allowed to become small as $\delta \rightarrow 0$, i.e., for exploring the possibility of distinguished limits arising between these two parameters. However, we expect the growth of perturbations with wavenumbers larger than these asymptotically small ones to be more relevant for the question of stability, and therefore will focus on them. We will verify the accuracy of asymptotic estimates derived from the WKB analysis by comparison with numerical results for the initial value problem (4.3) and will point out regions of small wavenumbers where we observe a reduction in accuracy.

Case $\boldsymbol{n}>\mathbf{3} / \mathbf{2}$. For $n=2$, the eigenvalue problem consisting of (4.14) and (4.19) was discussed and solved in [23], so we only briefly summarize the results. The essence of these results carries over to the case of general $n>3 / 2$. It turns out that for this problem we have two discrete eigenvalues that bifurcate out of the $\tilde{\lambda}=0$ eigenvalue at $\tilde{Q}=0$. The dispersion relation for the top eigenvalue, which is the important one for the question of instability, is shown in Figure 4(right). The long wave analysis for the top eigenvalue yields

$$
\phi_{1}=\partial_{\eta} \phi_{b}+\phi_{1,1}|\tilde{Q}|+O\left(\tilde{Q}^{2}\right), \quad \tilde{\lambda}=\tilde{\lambda}_{1}|\tilde{Q}|+O\left(\tilde{Q}^{2}\right),
$$

with $\tilde{\lambda}_{1}=2^{-1 / 2}$; see Appendix $\mathrm{C}$ where general expressions, considering $0 \leq n<3$, for $\tilde{\lambda}_{1}$ and $\phi_{1,1}$ are derived from an asymptotic long wave analysis. For the second discrete ("bottom") eigenvalue, the signs of the $O(\tilde{Q})$ corrections need to be reversed. As can be seen from Figure 4(right), the asymptotic result accurately captures the top eigenvalue obtained from the numerical computation for small $\tilde{Q}$ - in fact, up to $\tilde{Q}<1$.

4.3. Comparison of asymptotic and numerical solutions. For these comparisons, we confine ourselves to the values $n=0$ and $n=2$ in this section.

Case $\boldsymbol{n}=\mathbf{0}$. If we solve (4.3) for a fixed wavenumber $q$ and a "randomly chosen" initial perturbation (4.3f), we expect that for long times $t=\tau / \delta$, the solution is approximated by (4.4), (4.7), with the leading order behavior of $\Psi$ and $\sigma$ given in the subsequent derivations. We can check this by obtaining the solution to (4.3) numerically and verifying that

$$
\frac{d}{d t} \ln \left[\left\|\hat{h}_{1}(\cdot, t ; q)\right\|\right]=\delta^{4 / 5} \lambda(\tau ; Q)+O(1)
$$

where $\lambda$ is the eigenvalue obtained via (4.13) and (4.14), and $\|-\|$ is a convenient norm with respect to $x$. We will use the maximum norm. With (4.13), we can in fact write this as

$$
\frac{d}{d t} \ln \left[\left\|\hat{h}_{1}(\cdot, t ; q)\right\|\right]=\delta^{4 / 5} \dot{S}_{b, 0}^{4 / 3} \tilde{\lambda}(\tilde{Q})+O(\delta)
$$

or, applying $\dot{S}_{b, 0} \sim(2 / 5)^{3 / 5} \theta^{3 / 5}(\delta t)^{-3 / 5}$,

$$
\frac{d}{d t} \ln \left[\left\|\hat{h}_{1}(\cdot, t ; q)\right\|\right] \sim t^{-4 / 5}\left(\frac{2}{5}\right)^{4 / 5} \theta^{4 / 5} \tilde{\lambda}(\tilde{Q})+O\left(t^{-1}\right),
$$

with $q$ fixed. We can eliminate the explicit time dependence by using that $\max _{x} h_{b}=$ $\delta^{-1 / 5} \max _{X} H_{b, 0}$ and

$$
\max _{X} H_{b, 0}=\left(\frac{2}{5}\right)^{-1 / 5} \exp (-\pi / \sqrt{27}) \theta^{4 / 5} \tau^{1 / 5}+O\left(\delta^{1 / 5}\right),
$$

Copyright (c) by SIAM. Unauthorized reproduction of this article is prohibited. 

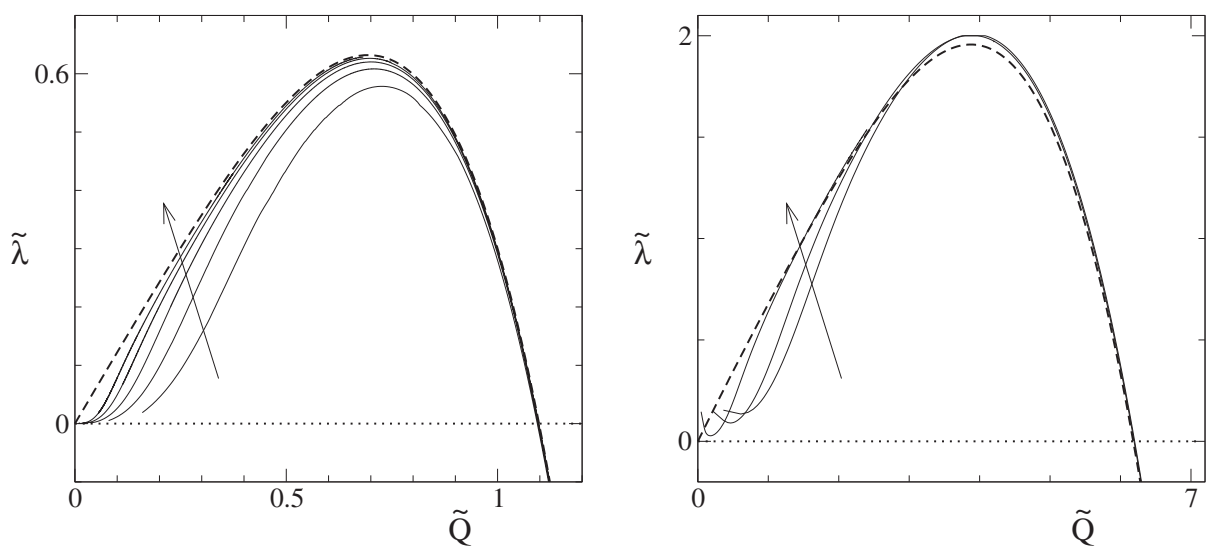

FIG. 5. Left: Comparison of the amplification rates of the solution to the linear stability problem (4.3) coupled to (3.1) with the dispersion relation $\tilde{\lambda}(\tilde{Q})$ for $n=0$. The dashed line is the top eigenvalue $\tilde{\lambda}(\tilde{Q})$ for (4.14) and (4.24) and is the same dispersion relation as in Figure 4(left). The solid lines are the graphs $\left(\left(1.831 \max _{x} h_{b}\right) q, \rho(q, t)\right)$ for amplification rates $\rho$ determined according to (4.28) for the numerical solutions of (4.3) coupled to (3.1) for fixed $q=1 / 16,1 / 32,1 / 64,1 / 128$, 1/256. The arrow points in the direction of decreasing $q$. Further details are given in the text. Right: Comparison for $n=2$. The dashed line is the dispersion relation as shown in Figure 4(right), and the solid lines are the amplification rates for $q=23.7 \times 10^{-3}, 14.1 \times 10^{-3}, 3.02 \times 10^{-3}$, obtained from numerical solutions of the linearized initial value problem for a regularized problem formulation as explained in the text. The arrow points in the direction of decreasing $q$.

and thus

$$
t^{1 / 5} \sim 1.831\left(\frac{2}{5}\right)^{1 / 5} \theta^{-4 / 5} \max _{x} h_{b}
$$

where we have evaluated the exponential expression. We choose $\theta=1$ and compare the graphs $\left(\left(1.831 \max _{x} h_{b}\right) q, \rho\right)$ and $(\tilde{Q}, \tilde{\lambda}(\tilde{Q}))$, where

$$
\rho(q, t) \equiv\left(1.831 \max _{x} h_{b}\right)^{4} \frac{d}{d t} \ln \left[\left\|\hat{h}_{1}(\cdot, t ; q)\right\|\right] .
$$

We expect agreement up to an error

$$
\rho(q, t)-\tilde{\lambda}(\tilde{Q})=O\left(\left(\max _{x} h_{b}\right)^{-1}\right),
$$

where

$$
\tilde{Q}=\left(1.831 \max _{x} h_{b}\right) q
$$

The results are shown in Figure 5. The solid lines with the values for $\rho$ in (4.28) were obtained from numerical solutions of (4.3) coupled to (3.1), while the dashed line with the graph for $\tilde{\lambda}$ is simply the dispersion relation from Figure 4 (left). It is clear that the graphs of $\rho$ for different $q$ converge to $\tilde{\lambda}$ as smaller $q$ are chosen, except for a region close to the origin. To understand the convergence, consider two different values for $q=q_{1}$ and $q=q_{2}$. These values map to $\left(1.831 \max _{x} h_{b}(x, t)\right) q_{1}$ and $\left(1.831 \max _{x} h_{b}(x, t)\right) q_{2}$, which coincide if we consider different times $t_{1}$ and $t_{2}$ so that $\max _{x} h_{b}\left(x, t_{1}\right) / \max _{x} h_{b}\left(x, t_{2}\right)=q_{2} / q_{1}$, that is, if the ridge is proportionally larger for the smaller wavenumber. This also means, according to (4.29), that the 
distance between $\rho\left(q_{1}, t_{1}\right)$ and $\rho\left(q_{2}, t_{2}\right)$ and the corresponding $\tilde{\lambda}(\tilde{Q})$ is proportionally smaller, for example, by a factor of two if $q_{1}$ and $q_{2}$ differ by a factor of two. This is approximately the case in the figure, except for very small $\tilde{Q}$. Moreover, for a fixed $q$, the value of $\max _{x} h_{b}(x, t)$ increases as we move to the right in Figure 5 , so all curves for $\rho$ are expected to approach the graph for $\tilde{\lambda}(\tilde{Q})$ (dashed curve) as $\tilde{Q}$ increases. which is indeed the case. The different behavior for small $\tilde{Q}$ is also expected in view of the discussion of the validity of the WKB analysis for small wavenumbers.

Case $\boldsymbol{n}=\mathbf{2}$. For $n=2$, similar derivations shows that the WKB result implies that

$$
\rho(q, t) \equiv\left(\frac{\max _{x} h_{b}}{\theta^{2} \max _{\xi} \phi_{b}}\right)^{2} \frac{d}{d t} \ln \left[\left\|\hat{h}_{1}(\cdot, t ; q)\right\|\right]
$$

where $\tilde{Q}=\left(\max _{x} h_{b} /\left(\theta \max _{\xi} \phi_{b}\right)\right) q$, must approach $\tilde{\lambda}(\tilde{Q})$ for fixed $q$ as $O\left(\left(\max h_{b}\right)^{-1}\right)$. We determine $\rho(q, t)$ from numerical solutions of a regularized thin film equation which includes an intermolecular potential to model the contact line region. This approach avoids a strict contact line at $x=s$ where $h=0$ by stabilizing a thin precursor of thickness $\nu \ll 1$, and the degeneracy of the PDE (1.1) leads to singularities in the solution. The sharp interface model with a fixed contact angle condition is recovered in the limit where the precursor thickness becomes small compared to the overall thickness of the film. Details of the model as well as the derivations and the numerical results have been given in $[23,35]$, so we only summarize them here.

The values of $\rho$ obtained from the solutions of the regularized problem are shown by solid lines in Figure 5(right), each one for a different choice of $q$. The dashed line is the dispersion relation for (4.14), (4.19), also shown in Figure 4(right). In the same fashion as for $n=0$, the graphs for $\rho$ converge to a single curve which is slightly larger than the dispersion relation. The deviation is on the order of the precursor thickness, which was chosen to be $\nu=0.04$. Also, as for $n=0$, the convergence is markedly slower for small values of $\tilde{Q}$.

4.4. Maximal amplification and dominant wavelength. We now would like to consider a superposition of modes with different wavenumbers, i.e.,

$$
h_{1}(x, y, t)=\int_{-\infty}^{\infty} \hat{h}_{1}(x, t ; q) \exp (i q y) d q,
$$

in order to determine the dominant wavenumber at a particular time $t$. Assuming that the rim is perturbed at a time $t_{0}$ by an initial perturbation

$$
h_{1, t_{0}}(x, y)=\int_{-\infty}^{\infty} \hat{h}_{1, t_{0}}(x, q) \exp (i q y) d q,
$$

where the initial amplitude of each contribution has a common fixed value for all wavenumbers, and applying (4.4) and (4.7) then results to leading order in

$$
h_{1}(x, y, t) \sim \int_{-\infty}^{\infty} \Psi_{0}(X, \tau ; Q) \exp \left(\int_{t_{0}}^{t} \lambda(\tau ; Q) d \tau\right) \exp (i Q y) d Q .
$$

The dominant wavenumber $Q_{d}$ at a given time $t$ is now the one for which the inner integral is maximal. Rewriting the integral according to (3.9) and taking derivatives with respect to $Q$ yields

$$
\int_{t_{0}}^{t} \dot{S}_{b, 0} \tilde{\lambda}^{\prime}\left(\tilde{Q}\left(\tau, Q_{d}\right)\right) d \tau=0
$$


Expressing the variables in $\tilde{Q}\left(\tau, Q_{d}\right)$ (for both cases $n=0$ and $\left.n>3 / 2\right)$ as

$$
\tilde{Q}\left(\tau, Q_{d}\right)=\frac{\rho_{n, \theta} Q_{d}}{c(\tau)}, \quad \rho_{n, \theta}=\theta^{n /(3-n)}, \quad c(\tau)=\dot{S}_{b, 0}^{1 /(3-n)}
$$

and observing that $\tilde{Q}$ is monotonically increasing in $\tau$, since $c(\tau) \sim \tau^{-1 /(5-n)}$, we may substitute $\tilde{Q}$ as the integration variable and obtain

$$
0=\int_{\tilde{Q}\left(t_{0}, Q_{d}\right)}^{\tilde{Q}\left(t, Q_{d}\right)}-\frac{c(\tau)^{5-n}}{\rho_{n, \theta} Q_{d} c_{\tau}(\tau)} \tilde{\lambda}^{\prime}(\tilde{Q}) d \tilde{Q} \sim \int_{\tilde{Q}\left(t_{0}, Q_{d}\right)}^{\tilde{Q}\left(t, Q_{d}\right)} \tilde{Q} \lambda^{\prime}(\tilde{Q}) d \tilde{Q} .
$$

Since $\tilde{Q}\left(t_{0}, Q_{d}\right)=\tilde{Q}\left(t, Q_{d}\right) c(t) / c\left(t_{0}\right) \rightarrow 0$ for $t \rightarrow \infty$, we can replace the lower integration limit by 0 and integrate (4.37) by parts to obtain for the asymptotic value of $\tilde{Q}=\tilde{Q}_{\infty} \equiv \lim _{t \rightarrow \infty} \tilde{Q}\left(t, Q_{d}\right)$ the relation

$$
\int_{0}^{\tilde{Q}_{\infty}} \tilde{\lambda}(\tilde{Q})-\tilde{\lambda}\left(\tilde{Q}_{\infty}\right) d \tilde{Q}=0
$$

The function $\tilde{\lambda}(\tilde{Q})$ is the time-independent dispersion relation, which we computed in section (4.2) for the cases $n=0$ and $n=2$, so we can determine the asymptotic wavenumber $\tilde{Q}_{\infty}=\tilde{Q}_{\infty}(n)$ from this result. Equation (4.38) has a simple interpretation in that is says that $\tilde{Q}_{\infty}$ is characterized as the value for which the area underneath the dispersion relation between zero and $\tilde{Q}_{\infty}$ is equal to the area of the rectangle with corners $(0,0)$ and $\left(\tilde{Q}_{\infty}, \tilde{\sigma}\left(\tilde{Q}_{\infty}\right)\right)$. The numerical values we find are $\tilde{Q}_{\infty}(0)=0.94$ and $\tilde{Q}_{\infty}(2)=5.3$, both rounded to two digits of accuracy.

The dominant wavenumber in the scalings of the growing rim is then $Q_{d}=$ $\tilde{Q}\left(t, Q_{d}\right) c(\tau) / \rho_{n, \theta}$. We compare this to an intrinsic length scale of the growing rim, for example, a suitably chosen measure of the width, $w(t)$. If the corresponding width for the outer solution is denoted by $\xi_{0}(n)$, then $w(t) \equiv \xi_{0}(n) \rho_{n, \theta} / c(\tau)$, and we obtain for the dominant wavelength $l_{d}(t)=2 \pi / Q_{d}(t)$ the expression

$$
\frac{l_{d}(t)}{w(t)}=\frac{2 \pi}{\xi_{0}(n) \tilde{Q}_{\infty}} .
$$

For $n>0$, the outer solution for the base state has two contact lines and it is natural to choose their distance for $\xi_{0}(n)=d_{b}$ so that, in particular for $n=2$, we have $\xi_{0}(2)=1 / 2$ and therefore $l_{d} / w=2.4$. For $n=0$, we choose the distance between the contact line at $\xi=0$ and the first zero crossing of (4.16), which gives $\xi_{0}(0)=3.63$, and then $l_{d} / w=1.8$. It is also instructive to compare the wavelength with the height of the rim $\max _{x} h_{b}$, which is less ambiguous, and this yields $l_{d} / \max _{x} h_{b}=14$ for $n=2$ and $l_{d} / \max _{x} h_{b}=12$ for $n=0$.

For $n=2$, the implications for experiments of these results on the dominant wavenumber was discussed in [34], so we focus here on the case $n=0$. The first question of interest is of course the maximum amplification achieved at the time when the base state ruptures. While it is difficult to set a specific threshold, it is clear that this amplification has to be significant for a clearly visible instability to occur while the rim is moving into the unperturbed film. The value of $q$ that will lead to the maximum amplification is the one that, upon rescaling as in (4.30), is equal to $\tilde{Q}_{d}$ determined above. Since the value of $\max _{x} h_{b}$ at the time of rupture was determined in section 3 following (3.16), we can find the estimate $q_{d}=1 / 15.5$. We 


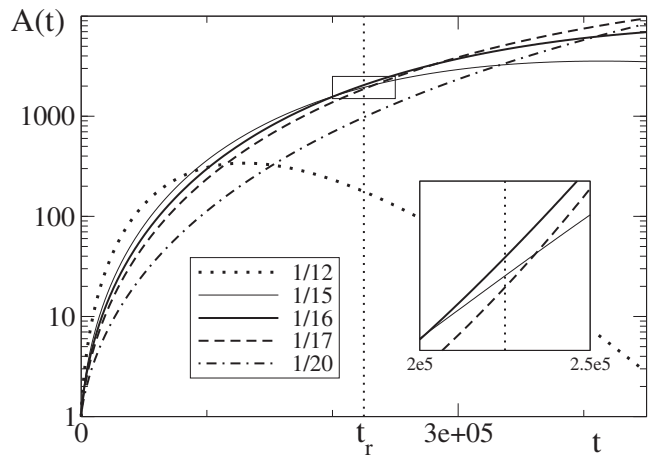

FIG. 6. Amplification of perturbations for $n=0$ for different wavenumbers $q$ as shown in the legends. For $q=12$, the amplification reaches a peak relatively early, and the period of decay leads to an amplification less than 1000 at the time $t=t_{r}$ where the base state ruptures (vertical dotted line; see also Figure 2). Conversely, for $q=1 / 20$, the perturbation is still growing and also has an amplification less than 1000 at $t=t_{r}$. The perturbation with the maximum amplification at $t=t_{r}$ has wavenumber $q=1 / 16$ (see inset, which is a zoom of the boxed region) and an amplification of about 2000. For $q<1 / 16$, a perturbation dominates at a specific time $t>t_{r}$, and conversely, $a$ perturbation with $q>1 / 16$ achieves dominance at at time $t<t_{r}$ but is then overtaken, in particular by the perturbation with $q=1 / 16$.

use this value and adjacent values of $q$ for our numerical solution for (4.3) and track the amplification

$$
A(t ; q)=\frac{\left\|\hat{h}_{1}(\cdot, t ; q)\right\|}{\left\|\hat{h}_{1, t_{0}}(\cdot ; q)\right\|}
$$

The result is shown in Figure 6. Among the wavenumbers used in Figure 6, the maximum amplification at $t=t_{r}$ is obtained for $q=1 / 16$, close to the estimate of $1 / 15.5$ given above for $q_{d}$.

For $q=1 / 16$, the numerical result gives an amplification of about 2000 at time $t=t_{r}$ when the base state ruptures and sheds material. The results in Figure 6 were achieved with a specific choice of the initial perturbation $\hat{h}_{1, t_{0}}$, which was set to be equal to the derivative of the initial profile for the base state (3.3). We tried other choices, which did not alter the dominant wavenumber at rupture time but did have an effect on the amplification rate. The reason for this is that while for the first choice of initial data the perturbation grew monotonically, there was an initial period of decay for the other choices. This indicates that the initial perturbation contained a higher fraction of modes that were stable and decayed, until finally the growth of the unstable mode dominated the evolution. The minimum was usually achieved early, at $t=10 \ldots 20$. To take this effect into account, we replaced the denominator in the definition of the amplification (4.40), and then the final amplification at $t=t_{r}$ was again close to the value of 2000 stated above.

It is remarkable that a suitable choice of scaling (4.13) leads to a fixed value $\tilde{Q}_{\infty}$ for the dominant wavenumber. This suggests that it could be advantageous to formulate the WKB ansatz (4.7) with $\sigma_{0}$ and $\tau$ that depend on $\tilde{Q}$ rather than on $Q$. This can be achieved by letting $\sigma_{0}(\tau, Q)=\tilde{\sigma}_{0}(\tau, \tilde{Q})$, where we have explicitly included the dependence on the wavenumber; we did this similarly for $\Psi$. The chain rule then implies that in the leading order problem, $\partial_{\tau} \sigma_{0}$ has to be replaced by 

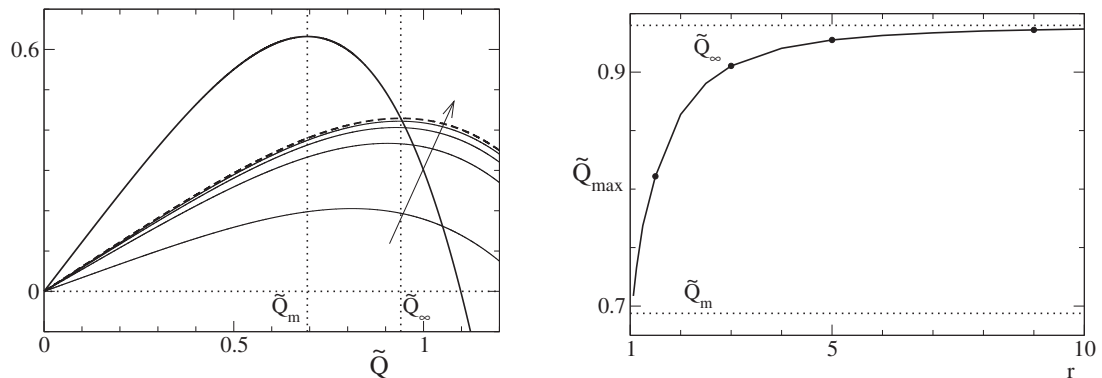

FIG. 7. Left: Dispersion relation $\tilde{\lambda}$ (thick solid line) compared to the amplification (except for prefactors) at large times $(r \rightarrow \infty)$, and $\Omega_{\infty}$ (dashed line) compared to the amplification at intermediate times, $\Omega(\tilde{Q}, r)$ with $r=1.5,3,5,9$ (thin solid lines). The arrow indicates increasing values of $r$. Right: Wavenumber with maximum amplification for different values of $r$.

$\partial_{\tau} \tilde{\sigma}_{0}-\left(\ddot{S}_{b, 0} / \dot{S}_{b, 0}(3-n)\right) \tilde{Q} \partial_{\tilde{Q}} \tilde{\sigma}_{0}$, so that $\tilde{\sigma}$ is determined from the PDE

$$
\partial_{\tau} \tilde{\sigma}_{0}-\frac{1}{3-n} \frac{\ddot{S}_{b, 0}}{\dot{S}_{b, 0}} \tilde{Q} \partial_{\tilde{Q}} \tilde{\sigma}_{0}=\dot{S}_{b, 0}^{\frac{4-n}{3-n}} \tilde{\lambda}(\tilde{Q})
$$

where $\tilde{\lambda}$ is given, as before, by (4.14). The first order PDE (4.41) requires initial conditions, and we assume that at some moment $\tau_{0}$, perturbations are present with amplification factor one for all wavenumbers $\tilde{Q}$, i.e., $\tilde{\sigma}_{0}\left(\tau_{0}, \tilde{Q}\right)=0$ for all $\tilde{Q}$. A more general situation could be considered, but this does not lead to an essentially different result. The solution is then given by

$$
\begin{aligned}
\tilde{\sigma}_{0} & =(5-n)\left(\frac{2 \alpha}{5-n}\right)^{\frac{4-n}{5-n}} \tau^{\frac{1}{5-n}} \Omega(\tilde{Q}, r), \\
\Omega(\tilde{Q}, r) & \equiv \frac{1}{\tilde{Q}}[\Lambda(\tilde{Q})-\Lambda(\tilde{Q} / r)], \\
\Lambda(\tilde{Q}) & \equiv \int_{0}^{\tilde{Q}} \tilde{\lambda}(z) d z,
\end{aligned}
$$

where $\alpha$ is shorthand for the prefactors in $(3.23)$ and $r \equiv\left(\tau / \tau_{0}\right)^{1 /(5-n)}$.

For $\tau \rightarrow \infty$, we have $r \rightarrow \infty$, and thus the logarithm of the amplification, $\tilde{\sigma}$, is proportional to

$$
\Omega_{\infty}=\frac{1}{\tilde{Q}} \int_{0}^{\tilde{Q}} \tilde{\lambda}(z) d z
$$

this is shown in Figure 7(left) by a dashed line and contrasted with the dispersion relation $\tilde{\lambda}(\tilde{Q})$. The maximum of the former curve is shifted compared to the dispersion relation, and in fact, it is equal to $\tilde{Q}_{\infty}$ determined earlier. Notice that $\tilde{\lambda}$ is the eigenvalue that would have been obtained from a frozen-mode analysis of the growing rim, and the comparison clearly shows that as time goes by (and $r$ increases), the most amplified wavenumber (in self-similar scales) shifts to larger values (shorter wavelengths). For intermediate values of $r, \Omega$ can be determined from (4.42b). For $r \rightarrow 1$, we recover $\tilde{\lambda}(\tilde{Q})$; for $r \rightarrow \infty$, the curve quickly converges to $\Omega_{\infty}$. Graphs for $\Omega$ are shown for different finite values of $r$ in Figure 7 (left). 
Correspondingly, the most amplified wavenumber $Q_{\max }$ of $\Omega$ increases from $Q_{m}$, i.e., the maximum for $\tilde{\lambda}$, to $Q_{\infty}$, determined by (4.38), as $r$ increases from one to infinity. In fact, convergence is nearly complete already for $r \geq 3$; see Figure 7(right). Because the maximum of the ridge scales like $S_{b, 0}^{1 / 2}$ and (3.23), the value $r$ can be interpreted as the ratio of the height of the rim at the current time divided by the height at the time when the perturbation is introduced. This implies that after a change in the height of the rim of a factor of three or more is observed as the instability develops, the most amplified wavenumber must be very close to the asymptotic value $\tilde{Q}_{\infty}$, much closer than to the value $\tilde{Q}_{m}$ predicted by the frozen-mode analysis.

5. Discussion and outlook. In this paper, we investigate the instability of unsteady and nonuniform base states for a free boundary problem of the thin film equation (1.1), specifically the retracting rim solutions that are susceptible to the "finger" instability. We develop a WKB method to address the multiscale aspects of the linear stability analysis that arise from the time dependence of the base states. The results are used to estimate the amplification of the perturbations and the wavenumber for which this amplification is maximal.

This wavenumber converges to a fixed value $\tilde{Q}_{\infty}$ scaled with the size of the growing rim, which is larger than the maximum wavenumber of the "frozen-mode" dispersion relation. It is interesting to note that, nevertheless, this value is determined by the dispersion relation through a simple expression (4.38). It would be interesting to see if this "shift" in the observed wavenumber can be detected in a physical experiment.

We note that the frozen-mode dispersion relation for the class of problems considered in this study has a linear behavior near $\tilde{Q}=0$, which is in contrast to the quadratic behavior found for the well-studied examples of fingering instabilities for gravity- or Marangoni-driven thin films [21,39].

For $n=0$, the evolution of the rim is halted by the rupture of the film, but, nevertheless, by the time the rupture occurs, the wavenumber has converged close to its asymptotic value. For $n=2$, the solution evolves into a spatially multilayered structure, which is readily accommodated within the WKB approach. The second value corresponds to the range $3 / 2<n<3$. A generalization of the approach, as well as some of the salient results, should carry over to the intermediate case $0<n \leq 3 / 2$ and also to appropriate formulations of $n \leq 3$, where in addition the contact line singularity needs to be addressed.

It would be fruitful to extend our method to a number of further problems with time-dependent base states such as the retracting soap films or surfactant-driven films. Moreover, for these and the problems studied in this work, it would also be interesting to investigate the influence of rim curvature in order to, for example, analyze the instabilities which appear in rims of dewetting circular holes.

We are currently considering aspects of the nonlinear stability via numerical simulations of (1.1), in particular the onset of finger pinch-off once they have grown sufficiently.

\section{Appendix A. Matching conditions for $3 / 2<n<3$.}

Base state. Let $w_{b}(t)$ be the width of the moving rim in (3.1), and let $w_{b}(t)=$ $\delta^{-1 /(5-n)} W_{b}(\tau)$ be the corresponding rescaled variable, according to the assumption that width and height of the rim scale identically. We define the boundary layer by

$$
x=\delta^{-1 /(5-n)} W_{b}(\tau)+\delta^{-\mu} \chi,
$$


with $\chi$ being the inner variable, where the scaling exponent $\mu$ remains to be determined. Apply (3.4) for $s_{b}$ while in the inner region $h_{b}$ is not rescaled, and consequently the far field condition remains

$$
\lim _{x \rightarrow \infty} h_{b}=1 .
$$

Applying (A.1) in (3.1a), we obtain

$$
\begin{gathered}
\delta \partial_{\tau} h_{b}-\delta^{(3-n) /(5-n)+\mu} \dot{S}_{b} \partial_{\chi} h_{b}-\delta^{(4-n) /(5-n)+\mu} \dot{W}_{b} \partial_{\chi} h_{b} \\
+\delta^{4 \mu} \partial_{\chi}\left(h_{b}^{n} \partial_{\chi \chi \chi} h_{b}\right)=0,
\end{gathered}
$$

suggesting the dominant balance $\mu=(3-n) /(3(5-n))$. Expanding $S_{b}$ as in (3.6) and $h_{b}, W_{b}$ analogously, i.e.,

$$
h_{b}(\chi, \tau ; \delta)=h_{b, 0}(\chi, \tau)+O\left(\delta^{1 /(5-n)}\right), \quad W_{b}(\tau ; \delta)=W_{b, 0}(\tau)+O\left(\delta^{1 /(5-n)}\right),
$$

yields the leading order problem

$$
-\dot{S}_{b, 0} \partial_{\chi} h_{b, 0}+\partial_{\chi}\left(h_{b, 0}^{n} \partial_{\chi \chi \chi} h_{b, 0}\right) .
$$

Integrating once and rescaling $\chi=\xi / \dot{S}_{b, 0}^{1 / 3}$ yields

$$
\left(h_{b, 0}-1\right)+h_{b, 0}^{n} \partial_{\xi \xi \xi} h_{b, 0}=0
$$

with solution

$$
h_{b, 0}(\xi) \sim\left(\frac{n^{3}}{3(3-n)(2 n-3)}(-\xi)^{3}\right)^{1 / n} \quad \text { for } \xi \rightarrow-\infty
$$

(in accordance with King and Bowen [22]). Hence, in outer coordinates we obtain the matching condition

$$
H_{b, 0}(X, \tau) \sim\left(\frac{n^{3}}{3(3-n)(2 n-3)}\right)^{1 / n} \dot{S}_{b, 0}^{1 / n}\left(W_{b, 0}(\tau)-X\right)^{3 / n}
$$

for $X \rightarrow W_{b, 0}(\tau)$.

Stability problem. Consider (4.3) and pass over to the inner layer analogously as in (A.6); i.e., rescale $x$ according to (A.1) and $s_{b}$ according to (3.4) while $h_{b}$ remains unscaled. Rescale $\hat{h}_{1}$ according to

$$
\hat{h}_{1}=\dot{s}_{b}^{1 / 3} \cdot w_{1} \cdot \hat{h}_{\mathrm{in}}=\delta^{-n /(3(5-n))} \dot{S}_{b}^{1 / 3} \cdot W_{1} \cdot \hat{h}_{\mathrm{in}},
$$

where $w_{1}$ denotes the perturbation of the second contact line and $W_{1}$ denotes the corresponding value in outer scalings. Note that this particular scaling results from matching both base state and perturbation. Moreover, apply the scales (4.4) for $q$ which altogether lead to the rescaled stability problem

$$
\begin{aligned}
0= & -\dot{S}_{b} \partial_{\chi} \hat{h}_{\mathrm{in}}+\partial_{\chi}\left[h_{b}^{n}\left(\partial_{\chi \chi \chi} \hat{h}_{\mathrm{in}}\right)+n\left(\partial_{\chi \chi \chi} h_{b}\right) h_{b}^{n-1} \hat{h}_{\mathrm{in}}\right]-\delta^{1 /(5-n)} \dot{W}_{b} \partial_{\chi} \hat{h}_{\mathrm{in}} \\
& +\delta^{4 n /(3(5-n))} Q^{4} h_{b}^{n} \hat{h}_{\mathrm{in}}-\delta^{2 n /(3(5-n))}\left[Q^{2} h_{b}^{n}\left(\partial_{\chi \chi} \hat{h}_{\mathrm{in}}\right)+\partial_{\chi}\left(Q^{2} h_{b}^{n} \partial_{\chi} \hat{h}_{\mathrm{in}}\right)\right] \\
& +\delta^{(3+n) /(3(5-n))}\left(\dot{S}_{b}^{1 / 3} \cdot W_{1}\right)^{-1} \partial_{\tau}\left(\dot{S}_{b}^{1 / 3} \cdot W_{1} \cdot \hat{h}_{\mathrm{in}}\right)
\end{aligned}
$$

Copyright (c) by SIAM. Unauthorized reproduction of this article is prohibited. 
Applying the asymptotic expansions (3.6) and (A.4), as well as

$$
\hat{h}_{\mathrm{in}}(\chi, \tau ; \delta)=\hat{h}_{\mathrm{in}, 0}(\chi, \tau)+O\left(\delta^{1 /(5-n)}\right), \quad W_{1}(\tau ; \delta)=W_{1,0}(\tau)+O\left(\delta^{1 /(5-n)}\right),
$$

for $\hat{h}_{1}$ and $W_{1}$, we obtain the leading order equation

$$
0=-\dot{S}_{b, 0} \partial_{\chi} \hat{h}_{\mathrm{in}, 0}+\partial_{\chi}\left[h_{b, 0}^{n}\left(\partial_{\chi \chi \chi} \hat{h}_{\mathrm{in}, 0}\right)+n\left(\partial_{\chi \chi \chi} h_{b, 0}\right) h_{b, 0}^{n-1} \hat{h}_{\mathrm{in}, 0}\right],
$$

which can be integrated once over $[\chi,+\infty)$,

$$
0=-\dot{S}_{b, 0} \hat{h}_{\mathrm{in}, 0}+h_{b, 0}^{n}\left(\partial_{\chi \chi \chi} \hat{h}_{\mathrm{in}, 0}\right)+n\left(\partial_{\chi \chi \chi} h_{b, 0}\right) h_{b, 0}^{n-1} \hat{h}_{\mathrm{in}, 0} .
$$

Rescaling $\chi=\xi / \dot{S}_{b, 0}^{1 / 3}$ (analogously as in (A.6)) reveals the ODE

$$
0=-\hat{h}_{\mathrm{in}, 0}+h_{b, 0}^{n}\left(\partial_{\xi \xi \xi} \hat{h}_{\mathrm{in}, 0}\right)+n\left(\partial_{\xi \xi \xi} h_{b, 0}\right) h_{b, 0}^{n-1} \hat{h}_{\mathrm{in}, 0}
$$

with solution

$$
\hat{h}_{\mathrm{in}, 0}(\xi) \sim 3 \cdot\left(\frac{n^{3-n}}{3(3-n)(2 n-3)}\right)^{1 / n} \cdot(-\xi)^{(3-n) / n} \quad \text { for } \xi \rightarrow-\infty .
$$

Matching into the outer problem thus requires $\mu_{2}=n /(3(5-n))$, and we obtain the outer matching condition

$$
\Psi_{0}(X, \tau) \sim 3 \cdot\left(\frac{-n^{3-n}}{3(n-3)(2 n-3)}\right)^{1 / n} \dot{S}_{b, 0}^{1 / n} \cdot W_{1,0} \cdot\left(W_{b, 0}(\tau)-X\right)^{(3-n) / n}
$$

for $X \rightarrow W_{b, 0}(\tau)$.

Appendix B. Roots of the characteristic polynomial. Consider

$$
f(z)=z^{4}-2 \tilde{Q}^{2} z^{2}-z+\tilde{Q}^{4}+\tilde{\lambda}
$$

where $z \in \mathbb{C}$ and $\tilde{Q} \geq 0$. We want to show that, if $\tilde{\lambda}$ is in the right half of the complex plane, then $f(z)=0$ has exactly two solutions with negative real part. Let $\tilde{\lambda}=\tilde{\lambda}_{r}+i \tilde{\lambda}_{i} \in \mathbb{C}$, with $\tilde{\lambda}_{i} \in \mathbb{R}$ and $\tilde{\lambda}_{r}>0$.

In the following we will exploit the argument principle in order to prove that $f(z)$ has two zeros in the left half plane, i.e., with negative real part. The argument principle states that, provided that there are no zeros on the boundary, the number of zeros of a polynomial inside a closed curve equals the change in argument over the curve divided by $2 \pi$. The closed curve which we would like to consider here is the left half circle of radius $R$ in the second and third quadrants (that is, start at zero, go along the positive imaginary axis until $R$, follow the circle of radius $R$ in the positive direction until the negative imaginary axis, and then return to the origin).

First, we check that there are no zeros on the boundary. For this purpose we consider $z=i x, x \in \mathbb{R}$, on the imaginary axis; $f(z)=0$ implies that

$$
x=\lambda_{i} \quad \text { and } \quad\left(x^{2}+\tilde{Q}^{2}\right)^{2}+\tilde{\lambda}_{r}=0,
$$

and both must be fulfilled, which is impossible since the left-hand side of the second equation is always positive; i.e., there are no zeros on the imaginary axis.

Copyright $@$ by SIAM. Unauthorized reproduction of this article is prohibited. 
We are now in position to apply the argument principle, which requires us to compute the change in argument of $f(z)$ in the three segments of our closed curve. For values $z=i x$ on the imaginary axis, we have

$$
f(i x)=\left(x^{2}+\tilde{Q}^{2}\right)^{2}+\tilde{\lambda}_{r}+i\left(\tilde{\lambda}_{i}-x\right),
$$

which always has positive real part so that we can use the formula

$$
\arg (f(i x))=2 \arctan \left(\frac{\tilde{\lambda}_{i}-x}{\sqrt{\left(\left(x^{2}+\tilde{Q}^{2}\right)^{2}+\tilde{\lambda}_{r}\right)^{2}+\left(\tilde{\lambda}_{i}-x\right)^{2}}+\left(x^{2}+\tilde{Q}^{2}\right)^{2}+\tilde{\lambda}_{r}}\right)
$$

to calculate the change in argument as $x$ takes values between 0 and $R$. For $x=0$ we consequently have $\arg (f(0))=0$, and as $R \rightarrow \infty$ we find $\arg (f(i R))=0$, which implies that the argument of $f(z)$ does not change on this part of the path.

On the circular arc, $z=R e^{i \theta}$, with $\pi / 2 \leq \theta \leq 3 / 2 \pi$, we have

$$
\begin{aligned}
f(z) & =R^{4} e^{4 i \theta}-2 \tilde{Q}^{2} R^{2} e^{2 i \theta}-R e^{i \theta}+\tilde{Q}^{4}+\lambda \\
& =R^{4} e^{4 i \theta}\left(1-\frac{2 \tilde{Q}^{2}}{R^{2} e^{2 i \theta}}-\frac{1}{R^{3} e^{3 i \theta}}+\frac{\tilde{Q}^{4}+\lambda}{R^{4} e^{4 i \theta}}\right),
\end{aligned}
$$

so as $R \rightarrow \infty$ we have $f(z)=R^{4} e^{4 i \theta}$, and as $\theta$ goes from $\pi / 2$ to $3 / 2 \pi$ the argument of $f(z)$ goes from $2 \pi$ to $6 \pi$, which gives a change in argument of $4 \pi$. Finally, the negative imaginary axis can be treated in a way analogous to the positive one, resulting in a change in argument of zero. In summary, as $R \rightarrow \infty$, the total change in argument is $4 \pi$, which implies that there are two zeros in the left half plane.

Appendix C. Long wave analysis. Consider the leading order eigenvalue problem (4.14) for $0 \leq n<3$ and introduce the expansions

$$
\begin{array}{r}
\phi_{1}(\xi, \tilde{Q})=\phi_{1,0}(\xi, \tau)+\tilde{Q} \phi_{1,1}(\xi, \tau)+\tilde{Q}^{2} \phi_{1,2}(\xi, \tau)+O\left(\tilde{Q}^{3}\right), \\
\tilde{\lambda}(\tilde{Q})=\tilde{\lambda}_{0}+\tilde{Q} \tilde{\lambda}_{1}(\tau)+\tilde{Q}^{2} \tilde{\lambda}_{2}(\tau)+O\left(\tilde{Q}^{3}\right) .
\end{array}
$$

Identifying the parts of $\tilde{\mathcal{L}}_{0}$ which are independent of and quadratic in $\tilde{Q}$ with $\tilde{\mathcal{L}}_{0,0}$ and $\tilde{\mathcal{L}}_{0,2}$, respectively, i.e.,

$$
\begin{aligned}
\tilde{\mathcal{L}}_{0,0} & \equiv(1-n) \partial_{\xi}-\left(\partial_{\xi} \phi_{b}^{n}\right) \partial_{\xi \xi \xi}-\phi_{b}^{n} \partial_{\xi \xi \xi \xi}, \\
\tilde{\mathcal{L}}_{0,2} & \equiv\left(\partial_{\xi} \phi_{b}^{n}\right) \partial_{\xi}+2 \phi_{b}^{n} \partial_{\xi \xi},
\end{aligned}
$$

we obtain from the leading order problem that $\tilde{\lambda}_{0}=0$ and $\phi_{1,0}=\partial_{\eta} \phi_{b}$. Proceeding to $O(\tilde{Q})$ and $O\left(\tilde{Q}^{2}\right)$ then reveals the problems

$$
\begin{aligned}
\tilde{\mathcal{L}}_{0,0} \phi_{1,1} & =\tilde{\lambda}_{1} \partial_{\xi} \phi_{b}, \\
\tilde{\mathcal{L}}_{0,0} \phi_{1,2}+\tilde{\mathcal{L}}_{0,2} \partial_{\xi} \phi_{b} & =\tilde{\lambda}_{1} \phi_{1,1}+\tilde{\lambda}_{2} \partial_{\xi} \phi_{b} .
\end{aligned}
$$

The $O(\tilde{Q})$ problem (C.3a) implies that $\phi_{1,1}$ must be the $\lambda_{1}$ multiple of the generalized eigenfunction of $\mathcal{L}_{0,0}$ for the eigenvalue $\lambda_{0}=0$ plus a multiple of $\Psi_{0,0}=\partial_{X} H_{b, 0}$, and we obtain

$$
\phi_{1,1}=-\frac{\tilde{\lambda}_{1}}{3-n}\left(\xi \partial_{\xi} \phi_{b}-\phi_{b}\right)
$$


and substituting this result into (C.3b) and integrating with respect to $\xi$ yields

$$
\int_{0}^{d_{b}} \tilde{\mathcal{L}}_{0,2} \partial_{\xi} \phi_{b} d \xi=-\frac{\tilde{\lambda}_{1}^{2}}{3-n} \int_{0}^{d_{b}} \xi \partial_{\xi} \phi_{b}-\phi_{b} d \xi
$$

for $0<n<3$ (note that contributions of $\tilde{\mathcal{L}}_{0,0} \phi_{1,2}$ and $\tilde{\lambda}_{2} \partial_{\eta} \phi_{b}$ vanish due to the boundary conditions for $\phi_{b}$ and $O\left(\tilde{Q}^{2}\right)$ boundary conditions for $\left.\phi_{1,2}\right)$ and

$$
\int_{0}^{\infty} \tilde{\mathcal{L}}_{0,0} \phi_{1,2} d \xi+\int_{0}^{\infty} \tilde{\mathcal{L}}_{0,2} \partial_{\xi} \phi_{b} d \xi=-\frac{\tilde{\lambda}_{1}^{2}}{3-n} \int_{0}^{\infty} \xi \partial_{\xi} \phi_{b}-\phi_{b} d \xi
$$

for $n=0$. In each case the integrals on both sides can be solved, and we obtain

$$
\tilde{\lambda}_{1}= \pm \sqrt{\frac{3-n}{2}} .
$$

Acknowledgment. The authors would like to thank Kevin Zumbrun for helpful advice and for pointing out the references $[29,30,42]$.

\section{REFERENCES}

[1] G. Agbaglah, C. Josserand, and S. Zaleski, Longitudinal instability of a liquid rim, Phys. Fluids, 25 (2013), 022103.

[2] A. L. Bertozzi and M. P. Brenner, Linear stability and transient growth in driven contact lines, Phys. Fluids, 9 (1997), pp. 530-539.

[3] A. L. Bertozzi, A. Münch, M. Shearer, and K. Zumbrun, Stability of compressive and undercompressive thin film travelling waves, European J. Appl. Math., 12 (2001), pp. 253291.

[4] F. Brochard-Wyart, P.-G. de Gennes, H. Hervert, and C. Redon, Wetting and slippage of polymer melts on semi-ideal surfaces, Langmuir, 10 (1994), pp. 1566-1572.

[5] F. Brochard-Wyart and C. Redon, Dynamics of liquid rim instabilities, Langmuir, 8 (1992), pp. 2324-2329.

[6] K. M. Butler And B. F. FARRell, Three-dimensional optimal perturbations in viscous shear flow, Phys. Fluids A, 8 (1992), pp. 1637-1651.

[7] J. W. Cahn and J. E. Taylor, Surface motion by surface diffusion, Acta Metall. Mater., 42 (1994), pp. 1045-1063.

[8] F. Doumenc, T. Boeck, B. Guerrier, And M. Rossi, Transient Rayleigh-Bénard-Marangoni convection due to evaporation: A linear non-normal stability analysis, J. Fluid Mech., 648 (2010), pp. 521-539.

[9] M. Dufay and O. Pierre-Louis, Anisotropy and coarsening in the instability of solid dewetting fronts, Phys. Rev. Lett., 106 (2011), 105506.

[10] B. D. Edmonstone, R. V. Craster, and O. K. Matar, Surfactant-induced fingering phenomena beyond the critical micelle concentration, J. Fluid Mech., 564 (2006), pp. 105-138.

[11] M. Ellero, X. Hu, J. Frohlich, and N. Adams, eds., Comprehensive Analysis of Dewetting Profiles to Quantify Hydrodynamic Slip, Springer, Dordrecht, 2009.

[12] J. C. Flitton and J. R. King, Surface-tension-driven dewetting of Newtonian and power-law fluids, J. Engrg. Math., 50 (2004), pp. 241-266.

[13] L. H. Gustavsson, Energy growth of three-dimensional disturbances in plane poiseuille flow, J. Fluid Mech., 224 (1991), pp. 241-260.

[14] M. G. Hennessy And A. MÜnch, Dynamics of a Slowly Evaporating Solvent-Polymer Mixture with a Deformable Upper Surface, OCCAM preprint 13/40, Mathematical Institute, University of Oxford, Oxford, UK, 2013.

[15] M. G. Hennessy And A. MÜNCh, A multiple-scale analysis of evaporation induced Marangoni convection, SIAM J. Appl. Math., 73 (2013), pp. 974-1001.

[16] D. S. Henningson, A. Lundbladh, and A. V. Johansson, A mechanism for bypass transition from localized disturbances in wall-bounded shear flows, J. Fluid Mech., 250 (1993), pp. 169207.

Copyright (c) by SIAM. Unauthorized reproduction of this article is prohibited. 
[17] K. H Hoffmann, B. Wagner, And A. MÜnch, On the generation and spreading of 'Finger' instabilities in film coating processes, in High Performance Scientific and Engineering Computing, Lecture Notes in Comput. Sci. Eng. 8, Springer, Berlin, 1999, pp. 245-254.

[18] C. Jahan, O. Faynot, L. Tosti, and J. M. Hartmann, Agglomeration control during the selective epitaxial growth of Si raised sources and drains on ultra-thin silicon-on-insulator substrates, J. Crystal Growth, 280 (2005), pp. 530-538.

[19] E. Jiran and C.V. Thompson, Capillary instabilities in thin, continuous films, Thin Solid Films, 208 (1992), pp. 23-28.

[20] W. Kan And H. Wong, Fingering instability of a retracting solid film edge, J. Appl. Phys., 97 (2005), 043515.

[21] D. E. Kataoka and S. M. Troian, A theoretical study of instabilities at the advancing front of thermally driven coating films, J. Colloid Interface Sci., 192 (1997), pp. 350 - 362.

[22] J. R. King And M. Bowen, Moving boundary problems and non-uniqueness for the thin film equation, European J. Appl. Math., 12 (2001), pp. 321-356.

[23] J. R. KING, A. MÜNCh, AND B. WAGNeR, Linear stability analysis of a sharp-interface model for dewetting thin films, J. Engrg. Math., 63 (2009), pp. 177-195.

[24] S. Kodambaka, J. Tersoff, M. C. Reuter, and F. M. Ross, Germanium nanowire growth below the eutectic temperature, Science, 316 (2007), pp. 729-732.

[25] F. Leroy, F. Cheynis, T. Passanante, and P. Müller, Dynamics, anisotropy, and stability of silicon-on-insulator dewetting fronts, Phys. Rev. B, 85 (2012), 195414.

[26] H. Lhuissier and E. Villermaux, The destabilization of an initially thick liquid sheet edge, Phys. Fluids, 23 (2011), 091705.

[27] W. Lick, The instability of a fluid layer with time-dependent heating, J. Fluid Mech., 21 (1965), pp. $565-576$.

[28] H. Machrafi, A. Rednikov, P. Colinet, and P.C. Dauby, Bénard instabilities in a binaryliquid layer evaporating into an inert gas, J. Colloid Interface Sci., 349 (2010), pp. 331-353.

[29] C. Mascia And K. Zumbrun, Stability of large-amplitude viscous shock profiles of hyperbolicparabolic systems, Arch. Ration. Mech. Anal., 172 (2004), pp. 93-131.

[30] C. Mascia And K. Zumbrun, Stability of small-amplitude shock profiles of symmetric hyperbolic-parabolic systems, Comm. Pure Appl. Math., 57 (2004), pp. 841-876.

[31] M. S. McCallum, P. W. Voorhees, M. J. Miksis, S. H. Davis, and H. Wong, Capillary instabilities in solid thin films: Lines, J. Appl. Phys., 79 (1996), p. 7604-7611.

[32] W. W. Mullins, Theory of thermal grooving, J. Appl. Phys., 28 (1957), pp. 333-339.

[33] A. Münch And B. Wagner, Contact-line instability of dewetting thin films, Phys. D, 209 (2005), pp. 178-190.

[34] A. MÜNCH AND B. WAGNER, Impact of slippage on the morphology and stability of a dewetting rim, J. Phys. Condens. Matter, 23 (2011), 184101.

[35] A. MÜnch, B. Wagner, And T. P. Witelski, Lubrication models with small to large slip lengths, J. Engrg. Math., 53 (2006), pp. 359-383.

[36] G. Reiter and A. Sharma, Auto-optimization of dewetting rates by rim instabilities in slipping polymer films, Phys. Rev. Lett., 87 (2001), 166103.

[37] L. B. Smolka and T. P. Witelski, On the planar extensional motion on an inertially driven liquid sheet, Phys. Fluids, 21 (2009), 042101.

[38] L. N. Trefethen, A. E. Trefethen, S. C. Reddy, and T. A. Driscoll, Hydrodynamic stability without eigenvalues, Science, 261 (1993), pp. 578-584.

[39] S. M. Troian, E. Herbolzheimer, S. A. Safran, and J. F. Joanny, Fingering instabilities of driven spreading films, Europhys. Lett., 10 (1989), pp. 25-30.

[40] M. R. E. Warner, R. V. Craster, and O. K. Matar, Unstable van der Waals driven line rupture in Marangoni driven thin viscous films, Phys. Fluids, 14 (2002), p. 1642.

[41] H. Wong, P. W. Vorhees, M. J. Miksis, and S. H. Davis, Periodic mass shedding of a retracting solid film step, Acta Mater., 48 (2000), pp. 1719-1728.

[42] K. Zumbrun and P. Howard, Pointwise semigroup methods and stability of viscous shock waves, Indiana Univ. Math. J., 47 (1998), pp. 741-872.

Copyright $@$ ㅇ by SIAM. Unauthorized reproduction of this article is prohibited. 